\title{
THE TRANSVERSE HOMOCLINIC DYNAMICS AND THEIR BIFURCATIONS AT NONHYPERBOLIC FIXED POINTS
}

\author{
BO DENG
}

\begin{abstract}
The complete description of the dynamics of diffeomorphisms in a neighborhood of a transverse homoclinic orbit to a hyperbolic fixed point is obtained. It is topologically conjugate to a non-Bernoulli shift called $\{\Sigma, \sigma\}$. We also obtain a more or less complete picture, referred to as the net weaving bifurcation, when the fixed point of such a system is undergoing the generic saddle-node bifurcation. The idea of homotopy conjugacy is naturally introduced to show that systems whose fixed points undergo the pitchfork, transcritical, periodic doubling, and Hopf bifurcations are all homotopically conjugate to our shift dynamics $\{\Sigma, \sigma\}$ in a neighborhood of a transverse homoclinic orbit. These bifurcations are also examined in the context of the spectral decomposition with respect to the maximal indecomposable nonwandering sets.
\end{abstract}

\section{INTRODUCTION}

Let $F: \mathbf{R}^{d} \rightarrow \mathbf{R}^{d}, d \geq 2$, be an invertible map and $F^{n}(\cdot):=F\left(F^{n-1}(\cdot)\right)$ the $n$th iterate map of $F$ for all $n \in \mathbf{Z}$. Then, by definition, the invariant set $\Lambda(F, U)$ of $F$ with respect to a given subset $U \subset \mathbf{R}^{d}$ is

$$
\Lambda(F, U):=\bigcap_{n \in \mathbf{Z}} F^{n}(U) .
$$

If the set $U$ contains a fixed point $q$ and a homoclinic orbit to the fixed point, $\gamma(p):=\left\{F^{n}(p): n \in \mathbf{Z}\right\}$, i.e., $F^{n}(p) \rightarrow q$ as $n \rightarrow \pm \infty$, then it must be the case that $\{q\} \cup \gamma(p) \subset \Lambda(F, U)$. Suppose $F$ is a $C^{r}$ diffeomorphism with $r \geq 1$. By reversing the iterate if necessary, one may always assume either $p \in W^{\text {cs }}-W^{\mathrm{u}}$ or

$$
p \in W^{\mathrm{cs}} \cap W^{\mathrm{u}},
$$

where $W^{c s}$ and $W^{\mathrm{u}}$ are the standard notation for center stable manifold and unstable manifold respectively. However, we are not interested in the former case because the invariant set $\Lambda$ must be entirely in the center stable manifold $W^{\text {cs }}$. Indeed, in this case $U$ does not intersect any global piece of the unstable manifold, thus $W^{\text {cs }}$ is attracting in $U$ and all the relevant dynamics associated with $\gamma(p)$ take place in a lower dimensional manifold $W^{\text {cs }}$. For this reason

Received by the editors May 31, 1989 and, in revised form, November 5, 1989.

1980 Mathematics Subject Classification (1985 Revision). Primary 34C28, 34C35, 34D30, $58 \mathrm{~F} 12,58 \mathrm{~F} 13,58 \mathrm{~F} 14,58 \mathrm{~F} 40$.

Key words and phrases. Transverse homoclinic point, saddle-node bifurcation, symbolic system, topological conjugacy. 


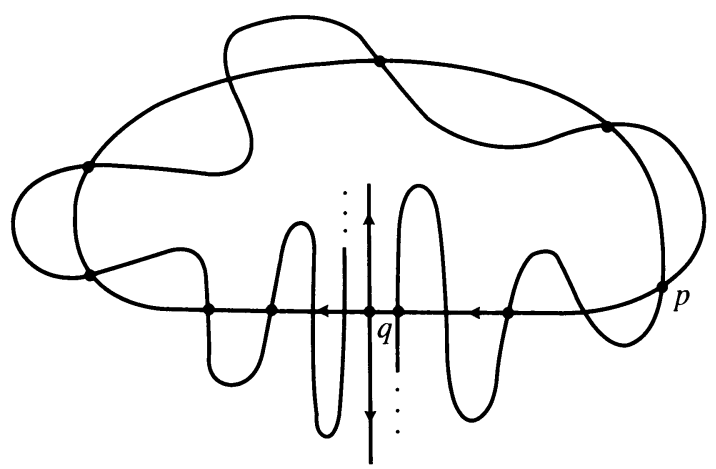

FIGURE 1.1

(1.1a) is referred to as the irreducible condition. Although the reducible homoclinic orbits are more common, the irreducible ones do appear in applications, as shown by an example at the end of this paper. Furthermore, at least in the case where $\mathbf{R}^{d}=\mathbf{R}^{2}$, the invariant set $\Lambda$ for an irreducible orbit is far more complex and interesting than that of a reducible one, which is simply the homoclinic orbit $\gamma(p)$ together with the fixed point $q$. See Figure 1.1. Therefore, our objective is to understand the dynamics, $\{\Lambda(F, U), F\}$, of such $F$ and $U$, where $U$ is a closed set and the interior, int $U$, contains an irreducible and transverse homoclinic orbit to a fixed point of hyperbolic or nonhyperbolic type. By transversality we mean the tangent spaces $T_{p} W^{\mathrm{cs}}, T_{p} W^{\mathrm{u}}$ at $p$ span the whole space

$$
T_{p} W^{\mathrm{cs}}+T_{p} W^{\mathrm{u}}=\mathbf{R}^{d} .
$$

The case with hyperbolic fixed point. This problem dates back to Poincare (1899) who realized that the presence of homoclinic orbits extremely complicates the dynamical structure. It was not until 1927 that the existence of countably many periodic points in $U$ had been proved by Birkhoff for the case where $q$ is a hyperbolic saddle point. In this case, of course, $p$ is always irreducible and $W^{\text {cs }}$ in the transverse condition $(1.1 \mathrm{~b})$ is understood as the stable manifold $W^{\text {s }}$. Thirty-six years later, Smale gave a better description by showing that there exist an integer $P$ and a closed set $R$ such that the $P$ th iterate $F^{P}$ on the invariant set $\Lambda\left(F^{P}, R\right)$ is topologically conjugate to the Bernoulli shift $\left\{\{1,2\}^{\mathbf{Z}}, \sigma\right\}$. Here $\{1,2\}^{\mathbf{Z}}$ is the space of all doubly infinite sequences of two symbols and the $i$ th component $(\sigma(s))_{i}$ of the image $\sigma(s)$ is defined as the $(i+1)$ th component $s_{i+1}$ of the preimage $s=\left(\cdots s_{-1} s_{0} s_{1} \cdots\right) \in\{1,2\}^{\mathbf{Z}}$. By topological conjugacy for two given dynamical systems $\{X, f\},\{Y, g\}$, we mean there is a homeomorphism $\rho: X \rightarrow Y$ such that $g \circ \rho=\rho \circ f$.

Illustrated in Figure 1.2 are two essentially different ways to construct the socalled Smale horseshoe map in $\mathbf{R}^{2}$ near a homoclinic orbit. The invariant set for the horseshoe (a) (cf., e.g., Smale (1963), Newhouse (1974), or Guckenheimer and Holmes (1983)) must be in $\Lambda(F, U)$ and contain the fixed point $q$ as well as the homoclinic point $p$, while that for (b) (cf., e.g., Wiggins (1988)) stays far away from $\Lambda(F, U)$. However, both of them are insufficient for understanding either the full dynamics of $\{\Lambda(F, U), F\}$ or its bifurcation. For instance, when the fixed point undergoes, say, a saddle-node bifurcation, in which case 


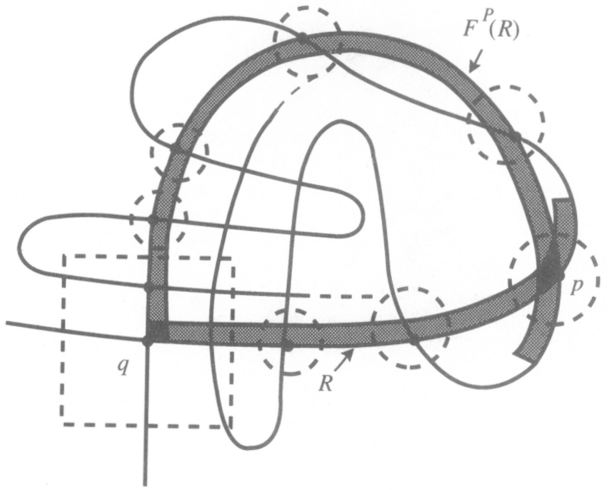

(a)

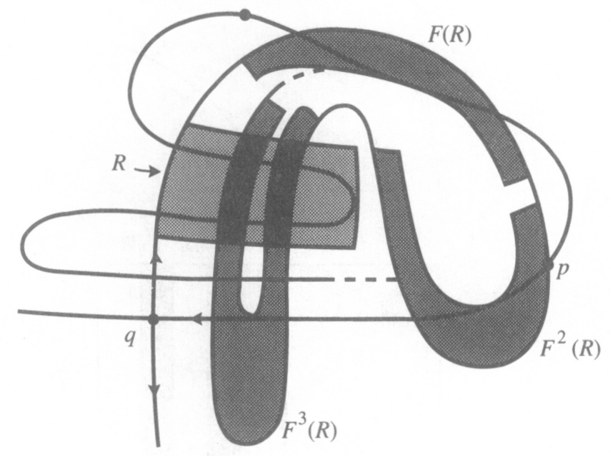

(b)

FIGURE 1.2

the fixed point as well as the homoclinic orbit may disappear altogether, the standard hyperbolic argument for the horseshoe dynamics will fail in case (a). In case (b), the horseshoe is likely to persist, but it is too far away from the homoclinic orbit to detect the ongoing bifurcation. Moreover, neither of them can tell for sure the existence of periodic orbits of those periods which are not the multiples of the iteration $P$. With regard to the second question, Sil'nikov obtained another not so well-known picture.

Theorem 1.1 (Sil'nikov, 1967). Let $F: \mathbf{R}^{d} \rightarrow \mathbf{R}^{d}$ be a diffeomorphism having a transverse homoclinic point $p$ to a hyperbolic fixed point $q$. Then, there exists a closed set $H$ whose interior int $H$ contains the homoclinic point $p$ such that the induced Poincaré map $\Pi: D(\Pi) \rightarrow H$ on the invariant set $\Omega:=\Lambda(\Pi, H)$ is topologically conjugate to the Bernoulli shift on the product space $\{1,2, \ldots\}^{\mathbf{Z}}$ of doubly infinite copies of the natural numbers.

Here, by definition, the domain $H_{0}:=D(\Pi)$ for the Poincaré return map $\Pi$ consists of those points $z$ in $H$ that have the first return iterate $z^{\kappa}:=$ $F^{\kappa}(z) \in H$ for some finite integer $\kappa:=\kappa(z)$. Of course, $\Pi(z):=z^{\kappa(z)}$. Theoretically speaking, the set of the natural numbers may be substituted by any countably infinite set. In particular, however, when $\{1,2,3, \ldots\}^{\mathbf{Z}}$ is replaced by $S:=\left\{K_{0}, K_{0}+1, \ldots\right\}^{\mathrm{Z}}$, where $K_{0}$ is the minimum iteration of points from $H_{0}$, i.e., $K_{0}=\min \left\{\kappa(z): z \in H_{0}\right\}$, then the symbolic system becomes more meaningful. Indeed, as will be shown later, the domain is decomposed as $H_{0}=\bigcup_{k \geq K_{0}} H_{0}^{k}$ with the property that each symbolically labelled (connected and closed) component $H_{0}^{k}$ consists of only those points $z$ so that $\kappa(z)=k$. As heuristically illustrated in Figure 1.3, the $H_{0}^{k}$ 's form a collection of "horizontal strands" while their images under the Poincaré return map form "vertical strands." We will loosely refer to this structure as the fishnet, in contrast to the Smale horseshoe.

In comparison with the horseshoe (a) in Figure 1.2, however, our fishnet is not flawless. Some crucial points, e.g., $p$ and $q$, are not in the net, not to mention the noncompactness of $\Omega$. Thus, to understand $\Lambda(F, U)$ better, we have to include the behavior of the dynamics on the stable and unstable manifolds in $H$. To do so, let $W_{H}^{\mathrm{s}}$ and $W_{H}^{\mathrm{u}}$ be the connected components of $W^{\mathrm{s}} \cap H$ and $W^{\mathrm{u}} \cap H$, respectively, containing the homoclinic point $p$. Note 


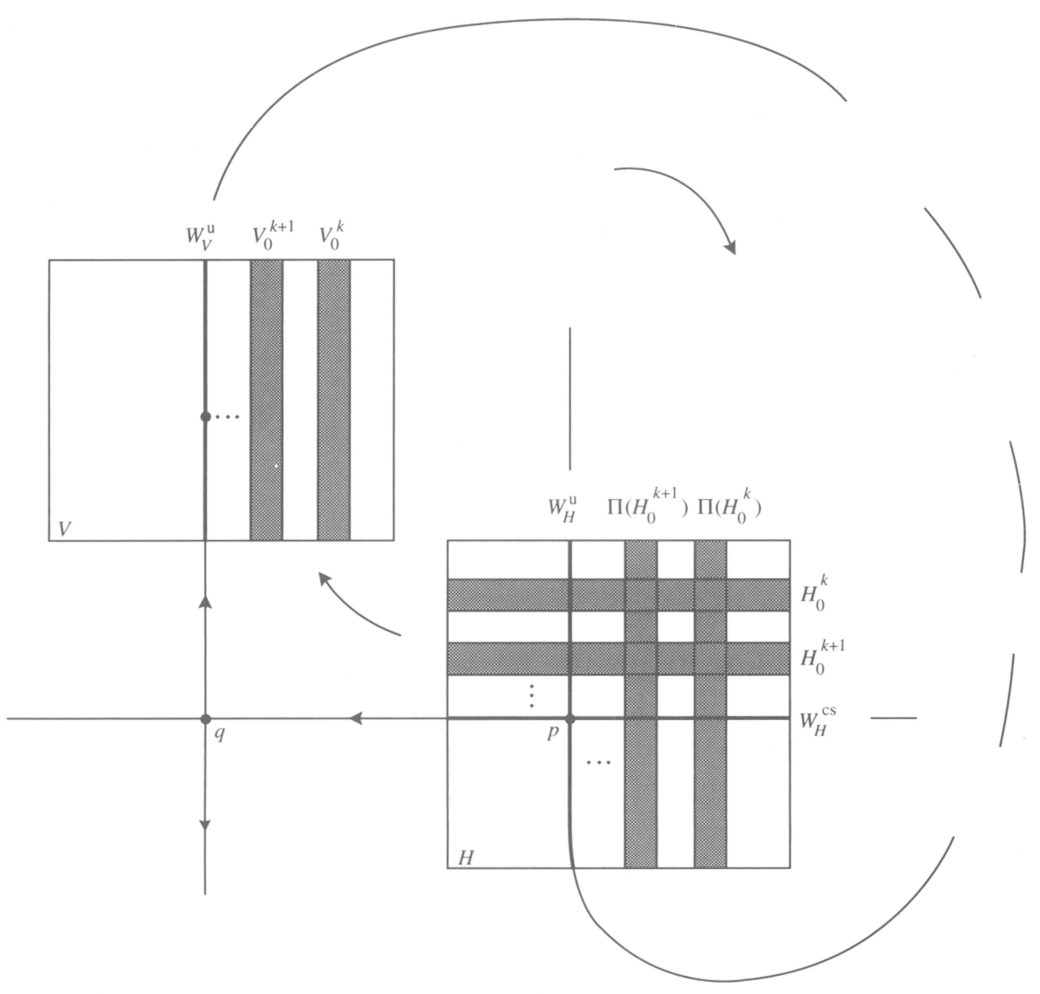

FIGURE 1.3

that, as suggested by Figure 1.2, the closure of our fishnet contains precisely $W_{H}^{\mathrm{s}}$ and $W_{H}^{\mathrm{u}}$ as the limit set. To continue, let $\Omega^{+} \subset H_{0}$ be the subset of those points $z \in H \cap W^{\text {s }}$ with the property that there exists an integer $n(z) \geq 0$ such that $\Pi^{k}(z) \in H_{0}$ are defined for all $-\infty<k \leq n-1$, and the $n$th iterate $\Pi^{n}(z) \in W_{H}^{\text {s }}$. That is, $z \in \Pi^{-n}\left(W_{H}^{\mathrm{s}}\right) \cap \bigcap_{k \leq n-1} \Pi^{-k}\left(H_{0}\right)$. Thus, as a set which is valid for all such possible $n \geq 0$, we have

$$
\Omega^{+}=\bigcup_{n \geq 0}\left\{\Pi^{-n}\left(W_{H}^{\mathrm{s}}\right) \cap\left(\bigcap_{k \leq n-1} \Pi^{-k}\left(H_{0}\right)\right)\right\} .
$$

Likewise, we define

$$
\Omega^{-}:=\bigcup_{m \leq 0}\left\{\Pi^{-m}\left(W_{H}^{\mathrm{u}}\right) \cap\left(\bigcap_{k \geq m+1} \Pi^{-k}\left(H_{0}\right)\right)\right\}
$$

which is the set of those points in $H$ whose forward iterates under $\Pi$ are all defined and fall into the unstable manifold $W_{H}^{\mathrm{u}}$ only after some finite backward iterates under $\Pi$. Similarly, $\Omega^{0}$ denotes the subset of homoclinic points to $q$ in $H$ such that $\Pi^{k}(z) \in H_{0}$ are defined for all $m+1 \leq k \leq n-1$ for every $z \in \Omega^{0}$ and for uniquely determined $m=m(z) \leq 0$ and $n=n(z) \geq 0$ with 
$\Pi^{n}(z) \in W_{H}^{\mathrm{s}}$ and $\Pi^{m}(z) \in W_{H}^{\mathrm{u}}$ respectively. In other words,

$$
\Omega^{0}=\bigcup_{\substack{m \leq 0 \\ n \geq 0}}\left\{\Pi^{-m}\left(W_{H}^{\mathrm{u}}\right) \cap\left(\bigcap_{k=m+1}^{n-1} \Pi^{-k}\left(H_{0}\right)\right) \cap \Pi^{-n}\left(W_{H}^{\mathrm{s}}\right)\right\} .
$$

Notice that the subsets $\left\{\Pi^{-n}\left(W_{H}^{\mathbf{s}}\right) \cap\left(\bigcap_{k \leq n-1} \Pi^{-k}\left(H_{0}\right)\right)\right\}$ in $\Omega^{+}$are mutually distinct for all $n \in \mathbf{Z}$ and for each fixed $n$ it equals the $n$th preimage of the same set $W_{H}^{\mathrm{s}} \cap\left(\bigcap_{k \leq-1} \Pi^{-k}\left(H_{0}\right)\right)$. The same observation also applies to $\Omega^{-}$ and $\Omega^{0}$. In regard to these sets, Sil'nikov has also proved

Theorem 1.2 (Sil'nikov, 1967). Assume the same condition as in Theorem 1.1. Then $\Omega^{i}, i \in\{+,-, 0\}$, is in one-to-one correspondence with $T^{i}$, where

$$
\begin{aligned}
T^{+} & :=\bigcup_{n \geq 0}\{1,2, \ldots\}^{\{\ldots, n-2, n-1\}}, \\
T^{-} & :=\bigcup_{m \leq 0}\{1,2, \ldots\}^{\{m, m+1, \ldots\}}, \\
T^{0} & :=\bigcup_{m \leq 0 \leq n}\{1,2, \ldots\}^{\{m, m+1, \ldots, n-1\}} .
\end{aligned}
$$

For the same reason as applied to $\Omega$ in Theorem 1.1, the set of the natural numbers $\{1,2, \ldots\}$ can be replaced by $\left\{K_{0}, K_{0}+1, \ldots\right\}$ so that the $T^{i}$ 's also become dynamically meaningful. Indeed, we will adopt this alternative interpretation from now on. Notice that the shift dynamics is not ready to be introduced to these sets, nor to the $\Omega^{i}$ 's by this theorem.

Except for the expression for the closure $\bar{\Omega}$ of $\Omega$, the following result is essentially due to Sil'nikov (1967) in the context of differential equations.

Theorem 1.3. Let $F: \mathbf{R}^{d} \rightarrow \mathbf{R}^{d}$ be a $C^{r}, r \geq 1$, diffeomorphism having a transverse homoclinic point $p$ to a hyperbolic fixed point $q$. Then, there exist closed sets $U$ and $H \subset U$ satisfying $\gamma(p) \cup\{q\} \subset$ int $U$ and $p \in$ int $H$ so that the closure

$$
\bar{\Omega}=\Omega \cup \Omega^{+} \cup \Omega^{-} \cup \Omega^{0},
$$

and

$$
\Lambda(F, U)=\overline{\bigcup_{n \in \mathbf{Z}} F^{n}(\boldsymbol{\Omega})}=\overline{\bigcup_{n \in \mathbf{Z}} F^{n}(\overline{\mathbf{\Omega}})}=\bigcup_{n \in \mathbf{Z}} F^{n}(\overline{\mathbf{\Omega}}) \cup\{q\},
$$

where $\Omega=\Lambda(\Pi, H)$, and $\Omega^{i}, i=0,+,-$, are defined as in (1.2a)-(1.2c).

This theorem says that $\Lambda(F, U)$ is basically generated by spreading $\bar{\Omega}$ around through the iterations of $F$ and exactly equals the resulting invariant set subject only to the one-point compactification. The added point is precisely the fixed point $q$.

Guided by this structure and the symbolic dynamics $\left\{S\left(K_{0}\right), \sigma\right\}$ for $\Pi$, we next derive the corresponding shift dynamical system, called $\{\Sigma, \sigma\}$, for $\{\Lambda, F\}$. The construction takes three steps. First let $\overline{\left\{k \geq K_{0}\right\}}:=\left\{K_{0}, K_{0}+\right.$ $1, \ldots\} \cup\{\infty\}$ denote the one-point compactification of $\left\{K_{0}, K_{0}+1, \ldots\right\}$ in the discrete topology, with $K_{0}$ arbitrary but fixed and $\overline{\left\{k \geq K_{0}\right\}} \mathbf{z}$ the corresponding doubly infinite product in the product topology. Select only the following 
subspaces $S=\left\{K_{0}, K_{0}+1, \ldots\right\}^{\mathrm{Z}}, T_{\infty}^{+}, T_{\infty}^{-}$, and $T_{\infty}^{0}$, where

$$
\begin{aligned}
& T_{\infty}^{+}:=\bigcup_{n \geq 0}\left\{s: s_{i} \geq K_{0}, i<n ; s_{j}=\infty, j \geq n\right\} \\
& T_{\infty}^{-}:=\bigcup_{m \leq 0}\left\{s: s_{i} \geq K_{0}, i \geq m ; s_{j}=\infty, j<m\right\} \\
& T_{\infty}^{0}:=\bigcup_{m \leq 0 \leq n}\left\{s: s_{i} \geq K_{0}, m \leq i<n ; s_{j}=\infty, \text { otherwise }\right\} .
\end{aligned}
$$

Namely, $T_{\infty}^{+}$is obtained from $T^{+}$by augmenting all the leftward infinite sequences into doubly infinite sequences with the symbol $\infty$ added. A similar explanation applies to $T_{\infty}^{-}$and $T_{\infty}^{0}$. Motivated by the roles of $W_{H}^{\mathrm{s}}$ and $W_{H}^{\mathrm{u}}$, which lie in the closure of the horizontal strands and the vertical strands respectively (cf. Figure 1.3), and a reason soon to be discussed we need to distinguish the symbol $\infty$ in $T_{\infty}^{+}$and $T_{\infty}^{-}$as $\infty^{\mathrm{s}}$ and $\infty^{\mathrm{u}}$ respectively. This leads to (1.3)

$$
\begin{aligned}
S^{+} & :=\bigcup_{n \geq 0}\left\{s: s_{i} \geq K_{0}, i<n ; s_{j}=\infty^{\mathrm{s}}, j \geq n\right\}, \\
S^{-} & :=\bigcup_{m \leq 0}\left\{s: s_{i} \geq K_{0}, i \geq m ; s_{j}=\infty^{\mathrm{u}}, j<m\right\}, \\
S^{0} & :=\bigcup_{m \leq 0 \leq n}\left\{s: s_{i} \geq K_{0}, m \leq i<n ; s_{j}=\infty^{\mathrm{s}}, j \geq n, s_{j}=\infty^{\mathrm{u}}, j<m\right\} .
\end{aligned}
$$

That is, $S^{i}$ is derived from $T_{\infty}^{i}$ by replacing all the leftward (rightward) infinite sequences of $\infty$ by leftward (rightward) sequences of $\infty^{\mathrm{u}}$ (while all the rightward ones by $\infty^{\mathrm{s}}$ ). We emphasize that $\infty^{\mathrm{s}}$ and $\infty^{\mathrm{u}}$ are treated as different symbols throughout and the introduction of $\overline{\left\{k \geq K_{0}\right\}} \mathbf{z}$ above is just a convenient way to describe the topology for the spaces $S, S^{+}, S^{-}$, and $S^{0}$. The necessity of using two symbols $\infty^{\mathrm{s}}, \infty^{\mathrm{u}}$ instead of $\infty$ alone may be best explained by the coding of the homoclinic orbit $\gamma(p)$. More precisely, the splitting of $\infty^{\mathrm{u}}$ and $\infty^{\mathrm{s}}$ at the $-n$th place in $\sigma^{n}\left(\cdots \infty_{1}^{\mathrm{u}} \cdot \infty_{0}^{\mathrm{s}} \infty_{1}^{\mathrm{s}} \cdots\right)$ is necessary in order to distinguish points $F^{n}(p)$ within the homoclinic orbit $\gamma(p)$. Without the superscripts all the homoclinic points would nonuniquely correspond to a single sequence.

We further clarify the somewhat mysterious topology of $S^{0}$. A typical topological basis element $B_{s_{m} \cdots s_{0} \cdots s_{n}}$ for a given $s \in S^{0}$, for example, satisfies the following. There may be some $j$ with $m \leq j \leq n$ such that $s_{j} \in\left\{\infty^{\mathrm{s}}, \infty^{\mathrm{u}}\right\}$. Then for such a $j$ there exists an integer $b_{j}$ with the property that the basis element consists of those sequences $s^{\prime}$ such that either $s_{i}^{\prime}=s_{i}$ for all $m \leq i \leq n$ or $s_{i}^{\prime}=s_{i}$ for all $m \leq i \leq n$, except for those $b_{j} \leq s_{j}^{\prime}<\infty$ with $s_{j} \in\left\{\infty^{\mathrm{s}}, \infty^{\mathrm{u}}\right\}$ and $s_{j}^{\prime} \neq s_{j}$.

In the second step, we will incorporate the meaning of $K_{0}$, the minimum returning iteration of all $z \in H_{0}$, into our symbolic system $\Sigma$. To do so, we need to 'blow up' or 'shift' the symbol $s_{i}$ according to the original dynamics $F$ rather than the Poincaré map. See Figure 1.4. When $s_{i} \neq \infty^{\mathrm{s}}, \infty^{\mathrm{u}}, s_{i}$ blows up into a unique string of $s_{i}$ copies of itself:

$$
\hat{s}_{i}:=s_{i}^{0} s_{i}^{1} \cdots s_{i}^{s_{i}-1},
$$




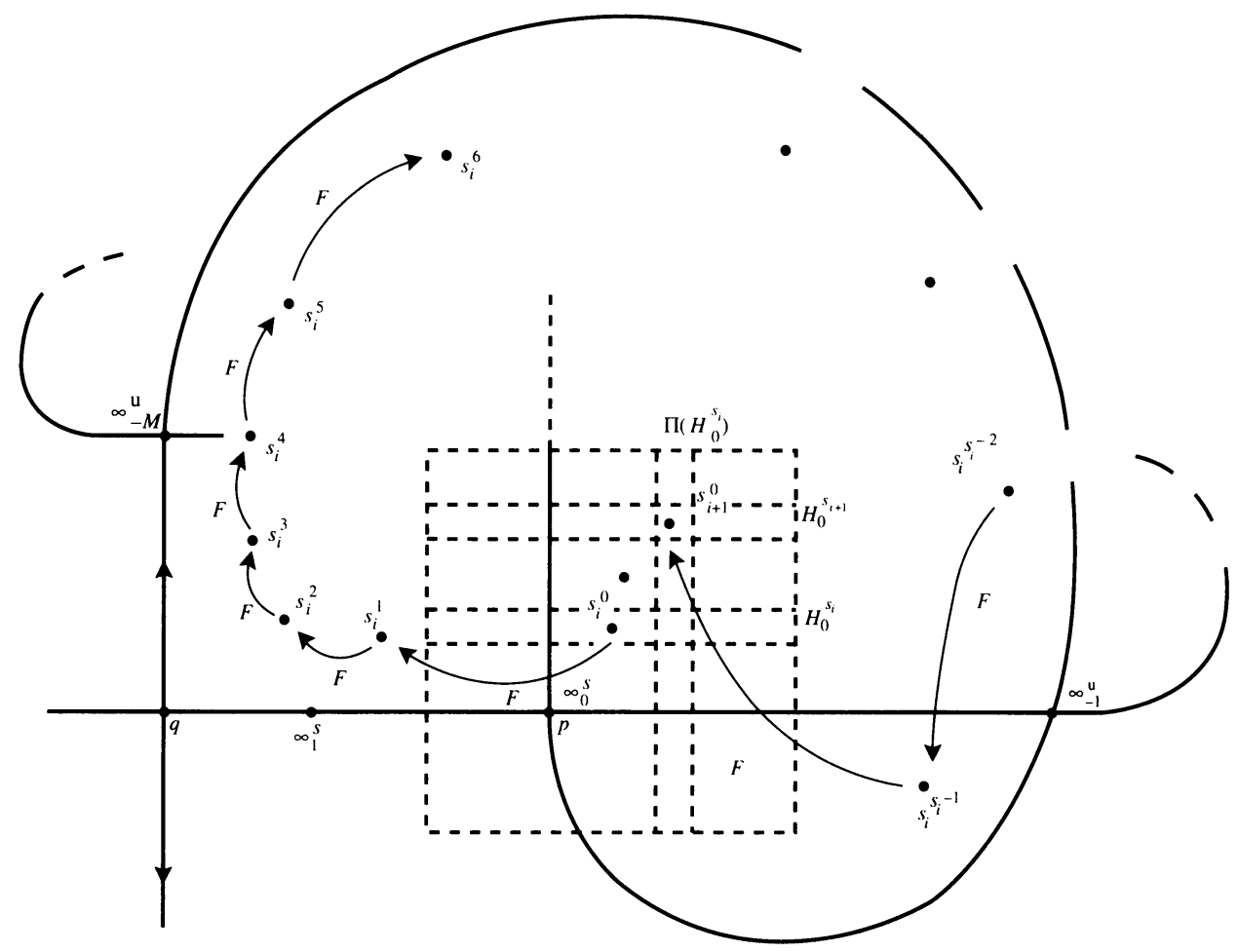

FIGURE 1.4

where, consistently, the superscripts are related to the iterate of the shift map $\sigma$ starting at $s_{i}^{0}$. When $s_{i}=\infty^{\mathrm{s}}$ or $\infty^{\mathrm{u}}, \hat{s}_{i}=s_{i}$, namely, the blow up of infinity is itself.

Let $\hat{S}$ and $\widehat{S}^{i}$ denote the corresponding sets of all the blowups. Parallel to the structure of $\Lambda(F, U)$, we also spread the standard blowups around by the shift operator and obtain $\bigcup_{n \in \mathbf{Z}} \sigma^{n}(\widehat{S}), \bigcup_{n \in \mathbf{Z}} \sigma^{n}\left(\widehat{S}^{i}\right)$ for $i \in\{+,-, 0\}$.

In the third step, we treat each set of the spread blowups as a subspace of $\overline{\left\{k \geq K_{0}\right\}^{\mathrm{Z}}}$ depending on its topology, treating $\infty^{\mathrm{s}}$ and $\infty^{\mathrm{u}}$ as distinct symbols. We then equip the union

$$
\bigcup_{n \in \mathbf{Z}} \sigma^{n}(\widehat{S}) \cup \bigcup_{n \in \mathbf{Z}} \sigma^{n}\left(\widehat{S}^{+}\right) \cup \bigcup_{n \in \mathbf{Z}} \sigma^{n}\left(\widehat{S}^{-}\right) \cup \bigcup_{n \in \mathbf{Z}} \sigma^{n}\left(\widehat{S}^{0}\right)
$$

with the topology generated by those of the spread blowups. It is easy to see that a description similar to the topology of $S^{0}$ above also applies to this generated spread topology. This will be made more precise later on when to deal with it extensively in the proof of our main result. For the time being, notice that the resulting space is not necessarily compact. For instance, the sequence $\left\{\left(\cdots \infty_{-1}^{\mathrm{u}} \infty_{0}^{\mathrm{u}} \cdots \infty_{n}^{\mathrm{u}} \infty_{n+1}^{\mathrm{s}} \cdots\right)\right\}_{n=1}^{\infty}$ of the homoclinic orbit $\gamma(p)$ on the local unstable manifold $W_{\text {loc }}^{\text {u }}$ does not have any limit point. Motivated by the structure of $\Lambda(F, U)$ of Theorem 1.3, we now complete our construction of $\Sigma\left(K_{0}\right)$ by taking the one-point compactification as follows:

$$
\Sigma:=\bigcup_{n \in \mathbf{Z}} \sigma^{n}(\widehat{\mathscr{S}}) \cup\{\omega\}
$$


Here,

$$
\mathscr{S}=S \cup S^{+} \cup S^{-} \cup S^{0}, \quad \widehat{\mathscr{S}}=\widehat{S} \cup \widehat{S}^{+} \cup \widehat{S}^{-} \cup \widehat{S}^{0},
$$

which are referred to as the standard sequences and the standard blowups respectively, and $\omega$ is the point added to the compactification that corresponds to the fixed point $\{q\}$ and it is defined as a fixed point of the extended shift map, namely, $\sigma(\omega):=\omega$. (One may denote $\omega=\left(\cdots \infty_{-1} \infty_{0} \infty_{1} \cdots\right)$.) One may also easily check that $\widehat{\mathscr{S}}$ is sequentially compact. This is not at all surprising since it will be shown later that $\widehat{\mathscr{S}}$ and $\bar{\Omega}$ are homeomorphic to each other. Note that the dependence of $\Sigma$ on $K_{0}$ is suppressed and will be so throughout so long as no confusion occurs. We now have the first of our main results.

Theorem 1.4. Assume the same conditions as in Theorem 1.3 and let $U, H$ be the same closed sets as in that theorem. Then the dynamical system $\{\Lambda(F, U)$, $F\}$ is topologically conjugate to the extended shift dynamics $\{\Sigma, \sigma\}$, where $\Sigma=$ $\Sigma\left(K_{0}\right)$ is defined as in (1.4) and $K_{0}=\min \left\{n: \Pi^{n}(z) \in H, z \in H_{0}\right\}$.

To distinguish among orbits, we call a periodic orbit $\gamma \quad l$-periodic if $\gamma \cap H_{0}$ contains only $l$ distinct points. We now have the following important properties for $\Lambda$.

Corollary 1.5. $\Lambda(F, U)$ is a Cantor set (i.e., compact, perfect, totally disconnected). It is chaotic in the usual sense, that is, it has

(a) a countable infinity of periodic orbits that is dense;

(b) an uncountable infinity of nonperiodic orbits;

(c) a dense orbit.

Moreover

(d) the set of homoclinic points to $q$ is countably infinite and is dense in $\Lambda$,

(e) there exists a unique 1-periodic orbit of every period $k \geq K_{0}$.

The proof of this corollary is trivial when one works with the symbolic system $\{\Sigma, \sigma\}$. Among other things, the properties (d) and (e) distinguish our dynamics $\{\Lambda, F\}$ from the horseshoe dynamics discussed in the beginning of this section. The 1-periodic orbits of large periods behave just like the shadow of the homoclinic orbit. They are expected to disappear together with the homoclinic orbit whenever the fixed point is perturbed away. They also serve as a criterion for how many symbols are actually needed by a symbolic description for the perturbed system. This will be made more precise when we deal with the nonhyperbolic fixed point case later. As another corollary to Theorem 1.4 we have

Theorem 1.6. $\{\Lambda, F\}$ is hyperbolic and structurally stable within the class of $C^{1}$ diffeomorphisms having a transverse homoclinic point to a hyperbolic fixed point.

The case with nonhyperbolic fixed point. Next, we consider the dynamical structure of $\Lambda_{\alpha}:=\Lambda(F(\cdot, \alpha), U)$ for a generic one-parameter family of $C^{r}, r \geq 4$, diffeomorphisms $F(\cdot, \alpha): \mathbf{R}^{d} \rightarrow \mathbf{R}^{d}, \alpha \in \mathbf{R}, d \geq 2 . F$ is also assumed $C^{r}$ in both $z$ and $\alpha$. We assume that $F$ has an irreducible and transverse homoclinic point $p$ to a nonhyperbolic fixed point $q$ at $\alpha=\alpha_{0}$. Let the closed sets $U$ and $H$ above be fixed for all the parameters below. Thus, all the definitions 
for the Poincaré map $\Pi$, its domain $H_{0}$, the subspaces $\Omega^{+}, \Omega^{-}, \Omega^{0}$, etc. can now be extended to the perturbed maps. They depend on the parameter $\alpha$ in general but the explicit dependence will be suppressed most of the time for simplicity of notation. For the necessary modification of the $\Omega^{i}$ 's, we only need to interpret $W^{\mathrm{s}}, W^{\mathrm{u}}$ in terms of the parametrically dependent $W^{\mathrm{cs}}(\alpha), W^{\mathrm{u}}(\alpha)$, in the definitions for $W_{H}^{\mathrm{s}}, W_{H}^{\mathrm{u}}$ and $(1.2 \mathrm{a})-(1.2 \mathrm{c})$ respectively.

In this introduction, we will only assume the fixed point to be a saddle-node point. That is,

1 is the only eigenvalue (counting algebraic multiplicity) of $D_{z} F\left(q, \alpha_{0}\right)$ on the unit circle of the complex plane.

Let $e_{l}$ and $e_{r}$ be a left and right eigenvector of 1 respectively. Then $e_{l} D_{z z} F\left(q, \alpha_{0}\right)\left(e_{r}, e_{r}\right) \neq 0$. More specifically, choose them in such a way that $e_{l} e_{r}=1$ and $e_{l} D_{z z} F\left(q, \alpha_{0}\right)\left(e_{r}, e_{r}\right)<0$.

The family of $F(\cdot, \alpha)$ is said to be generic if it satisfies

$$
\begin{aligned}
& e_{l} D_{\alpha} F\left(q, \alpha_{0}\right) \neq 0 . \text { More specifically, fix the direction } \alpha \text { in such } \\
& a \text { way that } e_{l} D_{\alpha} F\left(q, \alpha_{0}\right)>0 .
\end{aligned}
$$

Under this setting, the fixed point $q$ disappears when $\alpha<\alpha_{0}$, locally, while two hyperbolic fixed points, say $q_{+}$and $q_{-}$, bifurcate from $q$ when $\alpha>\alpha_{0}$, locally. Let $q_{+}$be the point for which $\operatorname{dim} W_{q_{+}}^{\text {s }}=\operatorname{dim} W_{q}^{\text {cs }}$. Thus, it is necessary that $\operatorname{dim} W_{q_{-}}^{\text {s }}=\operatorname{dim} W_{q}^{\text {s }}$. Here, $W_{q_{+}}^{\text {s }}$ means the stable manifold of $q_{+}$, etc. What has been described above can be obtained through the reduction of the map to the parametrically dependent center manifold $W^{\mathrm{c}}(\alpha)$. This has much in common with the spirit of Sotomayer (1974). As the last preparation, notice that due to the transversality condition (1.1b) and the implicit function theorem the irreducible 'homoclinic' point $p$ persists for all $\alpha$ near $\alpha_{0}$. Denote it by $p(\alpha)$. Then it is $C^{r}$ and satisfies $p\left(\alpha_{0}\right)=p$. In particular, when $\alpha \geq \alpha_{0}$, it becomes an irreducible, transverse homoclinic point to $q_{+}$. See Figure 1.5.

We now consider the structure and dynamics of $\Lambda_{\alpha}$. Recall $\Lambda_{\alpha}=$ $\Lambda(F(\cdot, \alpha), U)$. To motivate, we heuristically illustrate $\Lambda_{\alpha}$ with $d=2$ in Figure 1.5. This will help us foresee our main theorem for the general case.

We start with $\alpha=\alpha_{0}$. Intuitively, the fishnet structure is still there. But, less obviously, $\Lambda_{\alpha_{0}}$ is no longer totally disconnected. Indeed, one piece of the center stable manifold labelled $h_{p}$ in Figure 1.5(b) will remain in $U$ for all the forward and backward iterates of $F\left(\cdot, \alpha_{0}\right)$. Notice that its preimages are designated as $h_{p^{m}}$ in the picture and their length shrinks to zero as $m \rightarrow-\infty$, where $p^{m}=F^{m}(p)$. Actually, a closer examination on $\Omega^{0}$, the subset of homoclinic orbits to $q$, reveals the same behavior, that is, there is a short curve $h_{z} \subset W^{\text {cs }}$ rooted at every point $z \in \Omega^{0}$ such that $h_{z} \subset \Lambda_{\alpha_{0}}$. Surprisingly, however, this kind of fuzzy structure even extends to the entire subspace $\Omega^{-} \cup \Omega^{0}$, the set of unstable manifold in $\Lambda_{\alpha_{0}}$. But, as will be shown by the theorem below, this is the only additional feature attached to the net dynamics $\{\Sigma, \sigma\}$. We now move to $\alpha>\alpha_{0}$ where the two hyperbolic fixed points $q_{+}$and $q_{-}$split. The structure $\Lambda_{\alpha}$ is pretty much the same as $\Lambda_{\alpha_{0}}$ except the length of the hair $h_{z}$ approaches $\left|q_{+}-q_{-}\right|$as $z \rightarrow q_{+}$on the unstable manifold of $q_{+}$. See Figure 1.5(c). Last, when $\alpha<\alpha_{0}$, our fishnet with a countable infinity of horizontal and vertical strands together with the hairy structure are gone with the fish. 


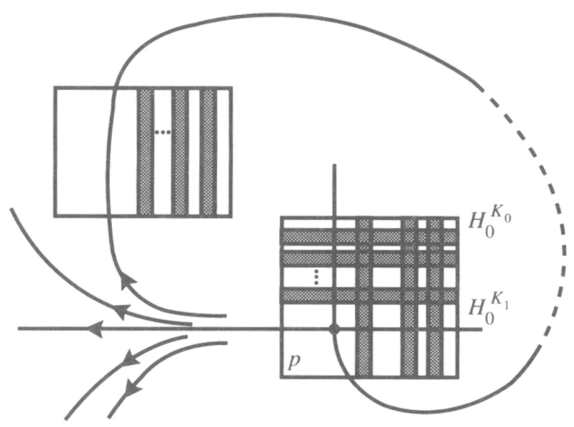

(a)

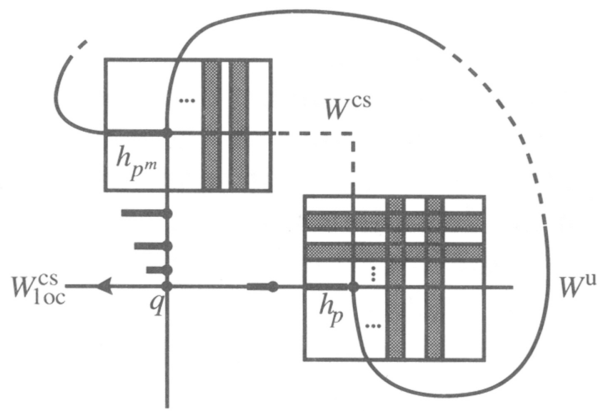

(b)

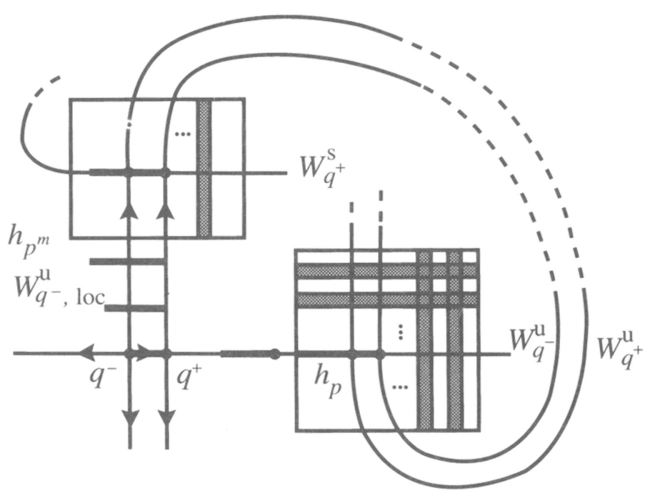

(c)

FIGURE 1.5

Instead, a torn net with only a finite number of strands is left. Repeating this scenario, but tracing $\alpha$ from left to right instead, we will achieve a reversal of the structure above. This is referred to as the net weaving bifurcation, which is now made precise by the following main result.

Theorem 1.7. Let $F(\cdot, \alpha): \mathbf{R}^{d} \rightarrow \mathbf{R}^{d}, \alpha \in \mathbf{R}, d \geq 2$, be a generic one-parameter family of $C^{r}, r \geq 4$, diffeomorphisms having an irreducible and transverse homoclinic point $p$ to a saddle-node fixed point $q$ at $\alpha=\alpha_{0}$, namely, conditions $(1.1 \mathrm{a}, \mathrm{b})$ and $(1.5 \mathrm{a}, \mathrm{b}, \mathrm{c})$ are satisfied. Then there exist closed sets $U$ and $H$ with $\gamma(p) \cup\{q\} \subset$ int $U, p \in$ int $H \subset$ int $U$, and a small number $\varepsilon_{0}$ such that the following holds for $\left|\alpha-\alpha_{0}\right|<\varepsilon_{0}$.

There exists a constant $K_{0}$ so that when $\alpha \geq \alpha_{0}$, the invariant set

$$
\bigcup_{n \in \mathbf{Z}} F^{n}(\bar{\Omega}, \alpha) \cup\left\{q_{+}\right\}
$$

is topologically conjugate to the shift dynamics $\left\{\Sigma\left(K_{0}\right), \sigma\right\}$ and

$$
\begin{aligned}
\Lambda(F(\cdot, \alpha), U)= & \bigcup_{n \in \mathbf{Z}} F^{n}(\bar{\Omega}, \alpha) \cup\left\{q_{+}\right\} \\
& \cup\left\{h_{z}: z \in \bigcup_{n \in \mathbf{Z}} F^{n}\left(\Omega^{-} \cup \Omega^{0}, \alpha\right)\right\}, \quad \alpha \geq \alpha_{0} .
\end{aligned}
$$

Here, $\Omega=\Lambda(\Pi, H), \Omega^{-}, \Omega^{0}$ are the same as in $(1.2 \mathrm{~b}, \mathrm{c})$ except that $W^{\mathrm{s}}$ is 
replaced by $W^{\mathrm{cs}}(\alpha)$, and each $h_{z}$ represents a $C^{r-3}$ curve homeomorphic to the unit close interval $[0,1]$ satisfying

(a) the collection of $h_{z}$ is invariant in the sense that $F\left(h_{z}, \alpha\right)=h_{F(z, \alpha)}$;

(b) $h_{z} \subset W^{\text {cs }}(\alpha)$ for $z \in \bigcup_{n \in \mathbf{Z}} F^{n}\left(\Omega^{0}\right)$;

(c) $h_{z}$ is the connected component in $\Lambda_{\alpha}$ containing $z$ and $h_{z^{\prime}} \cap h_{z}=\varnothing$ if $z^{\prime} \neq z$

(d) the length of $h_{z}$ approaches $\left|p_{+}(\alpha)-q_{-}(\alpha)\right|$ as $z \rightarrow q_{+}(\alpha)$ with $z \in W_{q_{+}}^{\mathrm{u}}$, where $q_{+}=q_{-}=q$ at $\alpha=\alpha_{0}$;

(e) every point from $h_{z}-\{z\}$, with $z \in \bigcup_{n \in \mathbf{Z}} F^{n}\left(\Omega^{0}, \alpha\right)$, is a reducible homoclinic point to $q$ at $\alpha=\alpha_{0}$ or a heteroclinic point from $q_{-}$to $q_{+}$for $\alpha>\alpha_{0}$.

In the case of $\alpha<\alpha_{0}$, there exists a constant $C_{0}>0$ independent of $\alpha$ so that $\Lambda_{\alpha}$ contains a subspace $\Omega^{*}(\alpha)$ with the property that the Poincare map $\Pi(\alpha)$ on $\Omega^{*}(\alpha)$ is topologically conjugate to the shift dynamics on doubly infinite sequences of finite symbols $\left\{K_{0}, K_{0}+1, \ldots, K_{1}(\alpha)\right\}$ with $K_{1}$ satisfying

$$
K_{1}(\alpha) \rightarrow \infty, \quad \text { as } \alpha \rightarrow \alpha_{0}^{-} \quad \text { and } \quad K_{1}(\alpha)<\frac{C_{0}}{\sqrt{\left|\alpha-\alpha_{0}\right|}} .
$$

Moreover, in contrast to $\left\{\Lambda_{\alpha_{0}}, F\left(\cdot, \alpha_{0}\right)\right\}$, there are no 1-periodic orbits of periods $\geq C_{0} / \sqrt{\left|\alpha-\alpha_{0}\right|}$ in $\Lambda_{\alpha}$.

The paper is organized as follows. In $\S 2$, we will establish two conditions which guarantee the existence of certain orbits of the Poincare map $\Pi$. In $\S 3$, we will prove our results (Theorems 1.1-1.6) for the hyperbolic fixed point case. This is done by checking the conditions of the main result, Lemmas 2.1-2.3, from $\S 2$. In $\S 4$, we will do very much the same thing as we do in $\S 3$ except that additional analysis on the local center manifold is carried out in order to apply those lemmas from $\S 2$. In $\S 5$, the concluding discussion, we will extend our main result to the transcritical, pitchfork, periodic doubling, and Hopf fixed points cases. We will also introduce the idea of homotopic conjugacy and show that the chaotic nature of $\Lambda_{\alpha}$ is best understood at this level of conjugacy. We will summarize our idea in proving the main theorems by a new proof of the classical example of Smale's horseshoe map, and derive a structural unstable horseshoe as well. Last, we will quote an example to which Theorem 1.7 immediately applies.

As a closing remark, we point out that Theorems 1.1-1.3 first appeared in Sil'nikov (1967) under the disguise of continuous flows. Moser (1973) apparently was not aware of Sil'nikov's works and independently discovered Theorem 1.1 through a more geometric approach.

\section{SOME TECHNICAL LEMMAS}

In this section, we will derive three technical lemmas which are applicable to both hyperbolic and nonhyperbolic fixed point cases. The first lemma is concerned with the doubly invariant set $\Omega$ while the second one is about, in a more general context, the forward and backward invariant sets in $H$ separately, including the behavior of the center stable manifold $W^{\text {cs }}$ and the unstable manifold $W^{\mathrm{u}}$ in $H$. To do so, we will establish an appropriate one-to-one correspondence between those sets with the fixed points of some systems of 'doubly' infinite, 'downward' infinite, or 'upward' infinite equations respectively. As a 
result, the fixed points are then parametrized by doubly, leftward, or rightward infinite sequences of countable symbols accordingly. While a doubly infinite sequence corresponds to a unique point in $\Omega$, a leftward or rightward one corresponds to a manifold which is a graph over $W_{\text {loc }}^{\text {cs }}$ or $W_{\text {loc }}^{\mathrm{u}}$ respectively. Lemma 2.3 , on the other hand, is concerned with the topological structure between the sequences and the invariant sets. It also gives a nice explanation as to why the blowup treatment to the symbols is necessary when the dynamics of the center stable manifold and the unstable manifold in $\Lambda$ are taken into consideration. Because of their generalities, the map $F$ here is always parametrically dependent, as are the invariant manifolds $W^{\mathrm{cs}}(\alpha)$ and $W^{\mathrm{u}}(\alpha)$. However, the parameter will be suppressed in our exposition most of the time. To begin with, we fix more notations for the rest of the paper.

Let us shift the fixed point $q$ at $\alpha=\alpha_{0}$ to the origin $q=0$ at $\alpha_{0}=$ 0 and choose $z=(x, y)$ as a $C^{r}$ local coordinate near the origin so that $W_{\text {loc }}^{\text {cs }}=\{y=0\}$ and $W_{\text {loc }}^{\mathrm{u}}=\{x=0\}$ locally. Such a normalization is standard by the $C^{r}$ smooth invariant manifold theory (see, e.g., Hirsch et al. (1977), Shub (1986), or Vanderbawhede and van Grils (1987)). Fix a closed $\delta$-box $B(\delta)=\{(x, y):|x| \leq \delta,|y| \leq \delta\}$ of the origin. For a given $\delta$, let $M=M(\delta)$ be the number of distinct points from the homoclinic orbit $\gamma(p)$ that lie outside the $\delta$-box. Obviously, it must be finite, satisfying $M \rightarrow \infty$ as $\delta \rightarrow 0$. Let $p_{0} \in \gamma(p) \cap W_{\mathrm{loc}}^{\mathrm{cs}} \cap B(\delta)$ and $p_{1} \in \gamma(p) \cap W_{\mathrm{loc}}^{\mathrm{u}} \cap B(\delta)$ be such that $p_{0}$ is the entering point on $\gamma(p)$ in the sense that $F^{-1}\left(p_{0}\right)$ is not in the box, while $p_{1}$ is the exiting point in the sense that $F\left(p_{1}\right) \notin B(\delta)$. Thus it must be $F^{M+1}\left(p_{1}\right)=p_{0}$. Certainly, one can easily manage to adjust $\delta$ so that both the entering and exiting homoclinic points are interior points of $B(\delta)$. This leads us to choose a closed $\delta_{i}$-box, $B\left(p_{i}, \delta_{i}\right)$, in $B(\delta)$ centered at $p_{i}$ for each $i=0$ and 1 respectively.

Now, for the consistency of notation, the domain of our return map will lie in the horizontal box

$$
H:=B\left(p_{0}, \delta_{0}\right),
$$

and for a reason soon to be clear the vertical box is

$$
V:=B\left(p_{1}, \delta_{1}\right) \text {. }
$$

The $\delta_{i}$ 's here are chosen so that $H$ and $V$ are iteratively disjoint in the sense that

$$
F^{i}(I) \cap I=\varnothing, \quad \text { for } i \in\{1,-1\} \text { and } I \in\{H, V\} .
$$

Moreover, we will also assume that for a given $\delta_{0}>0, \delta_{1}>0$ is chosen so that the image of $V$ under the $(M+1)$ th iterate falls into $H$ :

$$
F^{M+1}(V) \subset H .
$$

(The closed set $U$ will roughly be the same as $\bigcup_{i=1}^{M} F^{i}(V) \cup B(\delta)$. This will be made precise in the proof of our main theorem.)

The third constraint on the choice of $\delta_{0}$ and $\delta_{1}$ is related to the transverse intersection of the unstable manifold $W^{\mathrm{u}}$ and the center stable manifold $W^{\text {cs }}$ at $p_{0}$ and $p_{1}$. To be precise, let $\Pi_{g}:=F^{M+1}: V \rightarrow H$ be the global return map, and let $\left(\Pi_{g}^{x}, \Pi_{g}^{y}\right):=\Pi_{g}$ be its componentwise representation. To distinguish 
points between $H$ and $V$, we denote $\left(x_{\text {in }}, y_{\text {in }}\right) \in H$ while $\left(x_{\text {out }}, y_{\text {out }}\right) \in V$. Also, they are normalized so that

$$
\left(x_{\text {in }}, y_{\text {in }}\right)=0 \quad \text { at } p_{0} \quad \text { and } \quad\left(x_{\text {out }}, y_{\text {out }}\right)=0 \quad \text { at } p_{1} \text {. }
$$

See Figure 2.1 for the illustration. Thus, in terms of these local and normalized coordinates, the global maps read

$$
x_{\text {in }}^{j+1}=\Pi_{g}^{x}\left(x_{\text {out }}^{j}, y_{\text {out }}^{j}\right), \quad y_{\text {in }}^{j+1}=\Pi_{g}^{y}\left(x_{\text {out }}^{j}, y_{\text {out }}^{j}\right),
$$

where, consistently, the superscripts are included for keeping track of iterates later on and $\left|x_{\text {in }}^{j}\right|,\left|y_{\text {in }}^{j}\right| \leq \delta_{0}$ and $\left|x_{\text {out }}^{j}\right|,\left|y_{\text {out }}^{j}\right| \leq \delta_{1}$.

Now, recall the definitions $W_{H}^{\mathrm{cs}}$ and $W_{H}^{\mathrm{u}}$, which are the connected components of $W_{\mathrm{loc}}^{\mathrm{cs}}$ and $W^{\mathrm{u}}$ in $H$ containing the homoclinic point $p_{0}$. Thus, $W_{H}^{\text {cs }}:=\left\{\left(x_{\text {in }}, y_{\text {in }}\right): y_{\text {in }}=0,\left|x_{\text {in }}\right| \leq \delta_{0}\right\}$ and $W_{H}^{\mathrm{u}}=\Pi_{g}\left(W_{V}^{\mathrm{u}}\right)$, where symmetrically $W_{V}^{\mathrm{u}}:=\left\{\left(x_{\text {out }}, y_{\text {out }}\right): x_{\text {out }}=0,\left|y_{\text {out }}\right| \leq \delta_{1}\right\}$ (cf. Figure 2.1). In this context, the transversality condition $(1.1 \mathrm{~b})$ reads

$$
\operatorname{im} D_{y} \Pi_{g}(0,0)+\mathbf{R}^{d^{c s}} \times\{0\}=\mathbf{R}^{d},
$$

where $d^{\mathrm{cs}}$ and $d^{\mathrm{u}}$ with $d^{\mathrm{cs}}+d^{\mathrm{u}}=d$ are the dimensions of the center stable manifold and the unstable manifold respectively. Therefore, $D_{y} \Pi_{g}^{y}(0,0): \mathbf{R}^{d^{u}}$ $\rightarrow \mathbf{R}^{d^{\mathrm{u}}}$ must be nonsingular. Hence, by the implicit function theorem, $y_{\text {out }}^{j}$ can

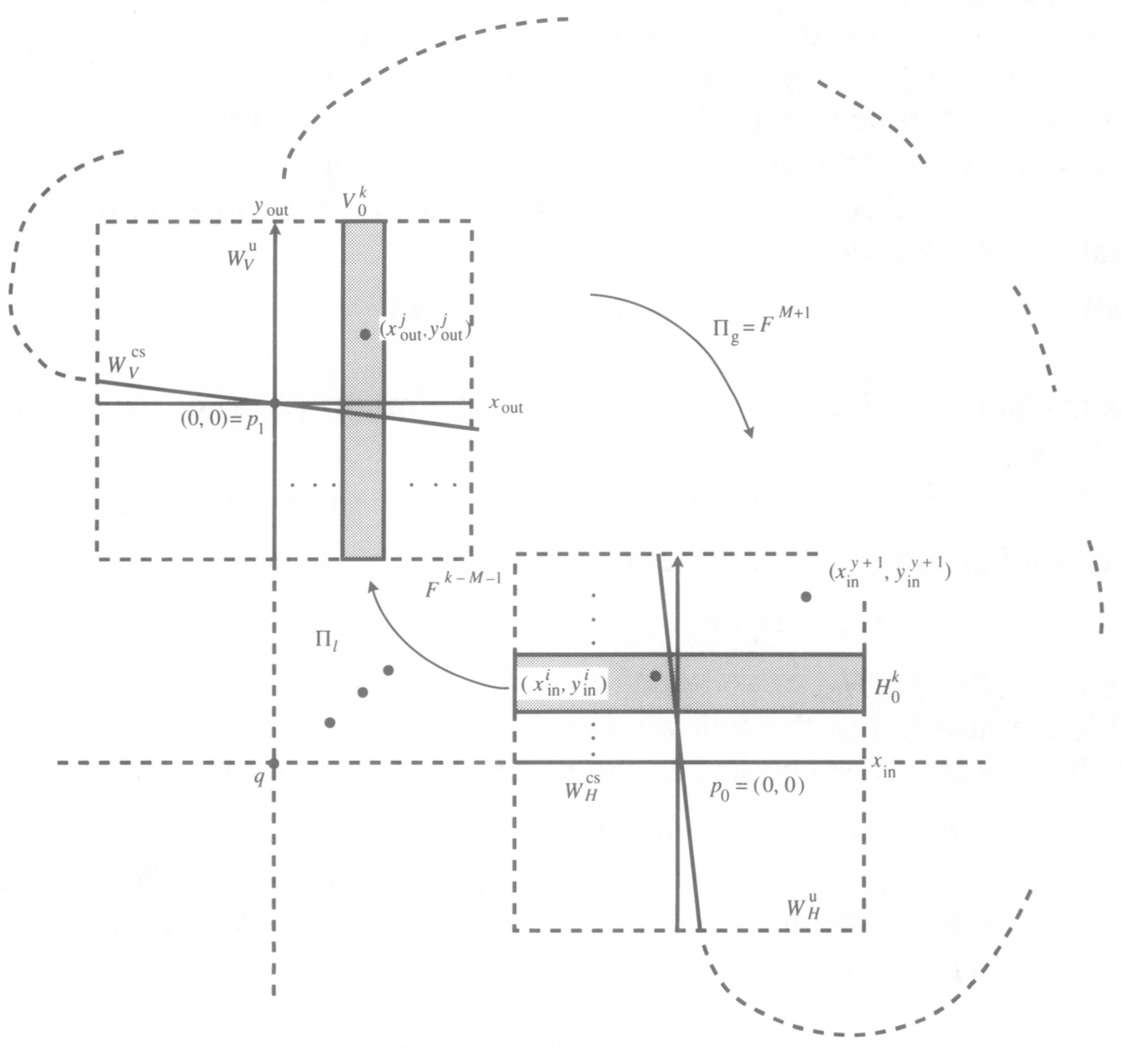

FIGURE 2.1 
be solved from the equation $y_{\mathrm{in}}^{j+1}=\Pi_{g}^{y}\left(x_{\text {out }}^{j}, y_{\text {out }}^{j}\right)$ in terms of the other two variables $x_{\text {out }}^{j}, y_{\text {in }}^{j+1}$ locally. Let

$$
y_{\text {out }}^{j}:=\widehat{\Pi}_{g}^{y}\left(x_{\text {out }}^{j}, y_{\text {in }}^{j+1}\right)
$$

denote the solution function; then it is easy to see that for given $\delta$ and $\delta_{0}, \delta_{1}$ can be chosen so small that, in addition to $(2.1 \mathrm{a}, \mathrm{b})$, the function $\widehat{\Pi}_{g}^{y}$ is defined for all $\left|x_{\text {out }}^{j}\right| \leq \delta_{1},\left|y_{\text {in }}^{j+1}\right| \leq \delta_{0}$, namely,

$$
\left|\widehat{\Pi}_{\mathrm{g}}^{y}\left(x_{\text {out }}^{j}, y_{\mathrm{in}}^{j+1}\right)\right| \leq \delta_{1}, \quad \text { for }\left|x_{\text {out }}^{j}\right| \leq \delta_{1},\left|y_{\text {in }}^{j+1}\right| \leq \delta_{0} .
$$

This basically concludes the choices of the constants $\delta, \delta_{0}$, and $\delta_{1}$. Let us now deliberately rewrite $(2.2 \mathrm{~b})$ as

$$
y_{\text {out }}^{j}=\widehat{\Pi}_{g}^{y}\left(x_{\text {out }}^{j}, y_{\text {in }}^{j+1}\right), \quad x_{\text {in }}^{j+1}=\Pi_{g}^{x}\left(x_{\text {out }}^{j}, \widehat{\Pi}_{g}^{y}\left(x_{\text {out }}^{j}, y_{\text {in }}^{j+1}\right)\right) .
$$

To define our return map $\Pi$ on $H$, we need to define a local return map $\Pi_{l}: D\left(\Pi_{l}\right) \rightarrow V$ so that $\Pi:=\Pi_{g} \circ \Pi_{l}$. By definition, the domain $D\left(\Pi_{l}\right) \subset H$ consists of those points $z \in H$ whose local orbits stay in $B(\delta)$ and exit the $\delta$-box of the origin only through the vertical $\delta_{1}$-box $V$ of $p_{1}$. In other words, there exists a $\kappa_{0}=\kappa_{0}(z)$ such that $F^{j}(z) \in B(\delta)$ for $0 \leq j \leq \cdot \kappa_{0}$, and $F^{j}(z) \in V$ when $j=\kappa_{0}$, Define

$$
N:=\left\{M+1+j: \exists z \in D\left(\Pi_{l}\right) \text { such that } j=\kappa_{0}(z)\right\},
$$

i.e., the subset of the natural numbers whose elements correspond to the first returning iterations of all points from $D\left(\Pi_{l}\right)$. It is important to note that $N$ depends not only on the choices of $\delta, \delta_{0}$, and $\delta_{1}$ but also on the parameter $\alpha, N=N\left(\delta, \delta_{0}, \delta_{1}, \alpha\right)$. As suggested by our Theorems 1.1 and 1.7 , the set $N$ is expected to be either $\left\{K_{0}, K_{0}+1, \ldots\right\}$ or just a finite set when $\alpha<\alpha_{0}$, or $\alpha<0$ in our normalized parameter. To continue, let $H_{0}^{k}$, in accordance with $\S 1$, be the subset of $D\left(\Pi_{l}\right)$ such that all the first returning iterations of $H_{0}^{k}$ equal $k \in N$. Hence,

$$
H_{0}:=D\left(\Pi_{l}\right)=\bigcup_{k \in N} H_{0}^{k} .
$$

It is trivial to conclude from the iterative disjointness (2.1a) of $H$ and $V$ and the closedness of $H$ that

(2.4c) $\quad H_{0}^{k} \cap H_{0}^{k^{\prime}}=\varnothing \quad$ if $k \neq k^{\prime}, k, k^{\prime} \in N \quad$ and $H_{0}^{k}$ are closed.

Next, similar to the global representation $(2.2 \mathrm{~b})$, we write

$$
x_{\text {out }}^{j}=\Pi_{l}^{x}\left(x_{\text {in }}^{j}, y_{\text {in }}^{j}\right), \quad y_{\text {out }}^{j}=\Pi_{l}^{y}\left(x_{\text {in }}^{j}, y_{\text {in }}^{j}\right),
$$

where $\left(x_{\text {in }}^{j}, y_{\text {in }}^{j}\right) \in H_{0}$.

We are now led to the following crucial condition for our Lemmas 2.1 and 2.2. It is meant to be checked in the proofs of our main theorems later on.

(2.5b) There are Lipschitz functions $\hat{x}\left(k, x_{\text {in }}^{j}, y_{\text {out }}^{j}\right), \hat{y}\left(k, x_{\text {in }}^{j}, y_{\text {out }}^{j}\right)$ of all $\left|x_{\text {in }}^{j}\right| \leq \delta_{0}$ and $\left|y_{\text {out }}^{j}\right| \leq \delta_{1}$ for each $k \in N$ such that the representation (2.5a) for the local map is equivalent to the cross representation

$$
x_{\text {out }}^{j}=\hat{x}\left(k, x_{\text {in }}^{j}, y_{\text {out }}^{j}\right), \quad y_{\text {in }}^{j}=\hat{y}\left(k, x_{\text {in }}^{j}, y_{\text {out }}^{j}\right)
$$

for all $\left|x_{\text {in }}^{j}\right| \leq \delta_{0},\left|y_{\text {out }}^{j}\right| \leq \delta_{1}$. 
Throughout, $\Delta:=\left\{\zeta^{j}=\left(x_{\text {in }}^{j}, y_{\text {out }}^{j}\right):\left|x_{\text {in }}^{j}\right| \leq \delta_{0}\right.$ and $\left.\left|y_{\text {out }}^{j}\right| \leq \delta_{1}\right\}$ is called the Sil'nikov domain and $\zeta^{j}$ the Sil'nikov variable. Note that $\Delta$ is a rectangular closed set in $\mathbf{R}^{d}$ and is independent of the first returning iteration $k$ of points in $H_{0}^{k}$. The virtue of such a hypothetical (at this moment) representation $(2.5 \mathrm{~b})$ is to allow us to treat those otherwise less tractable variables $x_{\text {in }}^{j}$ and $y_{\text {out }}^{j}$ as independent variables. As the last two pieces of terminology, we have

$$
\theta_{\text {in }}^{k}:=\left(x_{\text {in }}^{j}, \hat{y}\left(k, x_{\text {in }}^{j}, y_{\text {out }}^{j}\right)\right), \quad \theta_{\text {out }}^{k}:=\left(\hat{x}\left(k, x_{\text {in }}^{j}, y_{\text {out }}^{j}\right), y_{\text {out }}^{j}\right),
$$

for $\left|x_{\text {in }}^{j}\right| \leq \delta_{0},\left|y_{\text {out }}^{j}\right| \leq \delta_{1}$. The former is referred to as the Sil'nikov change of variables while the latter is the local map on $H_{0}^{k}$ in the new variable. See Figure 2.2.

Having obtained the two key ingredients $(2.3)$ and $(2.5 b)$ of this section, we are now in a position to consider the orbit $\gamma\left(z^{0}\right)=\left\{z^{j}=\Pi^{j}\left(z^{0}\right): j \in \mathbf{Z}\right\}$ through a point $z^{0} \in \Omega$. Recall $\Omega=\bigcap_{n \in \mathbf{Z}} \Pi^{n}\left(H_{0}\right)$, the invariant set of $\Pi$ in $H_{0}$. Such an orbit uniquely gives rise to a doubly infinite sequence $s=\left(\cdots s_{-1} s_{0} s_{1} \cdots\right)$ with the property that the $j$ th iterate $z^{j}=\left(x_{\text {in }}^{j}, y_{\text {in }}^{j}\right)$ belongs to $H_{0}^{s_{j}}$ for a uniquely determined $s_{j} \in N$ since $H_{0}^{k}$ is disjoint for distinct $k$ by $(2.4 \mathrm{c})$. Now, substituting the Sil'nikov change of variable $(2.5 \mathrm{~b})$ into the equation (2.3) above and appending the equations with all the $j \in \mathbf{Z}$, we are
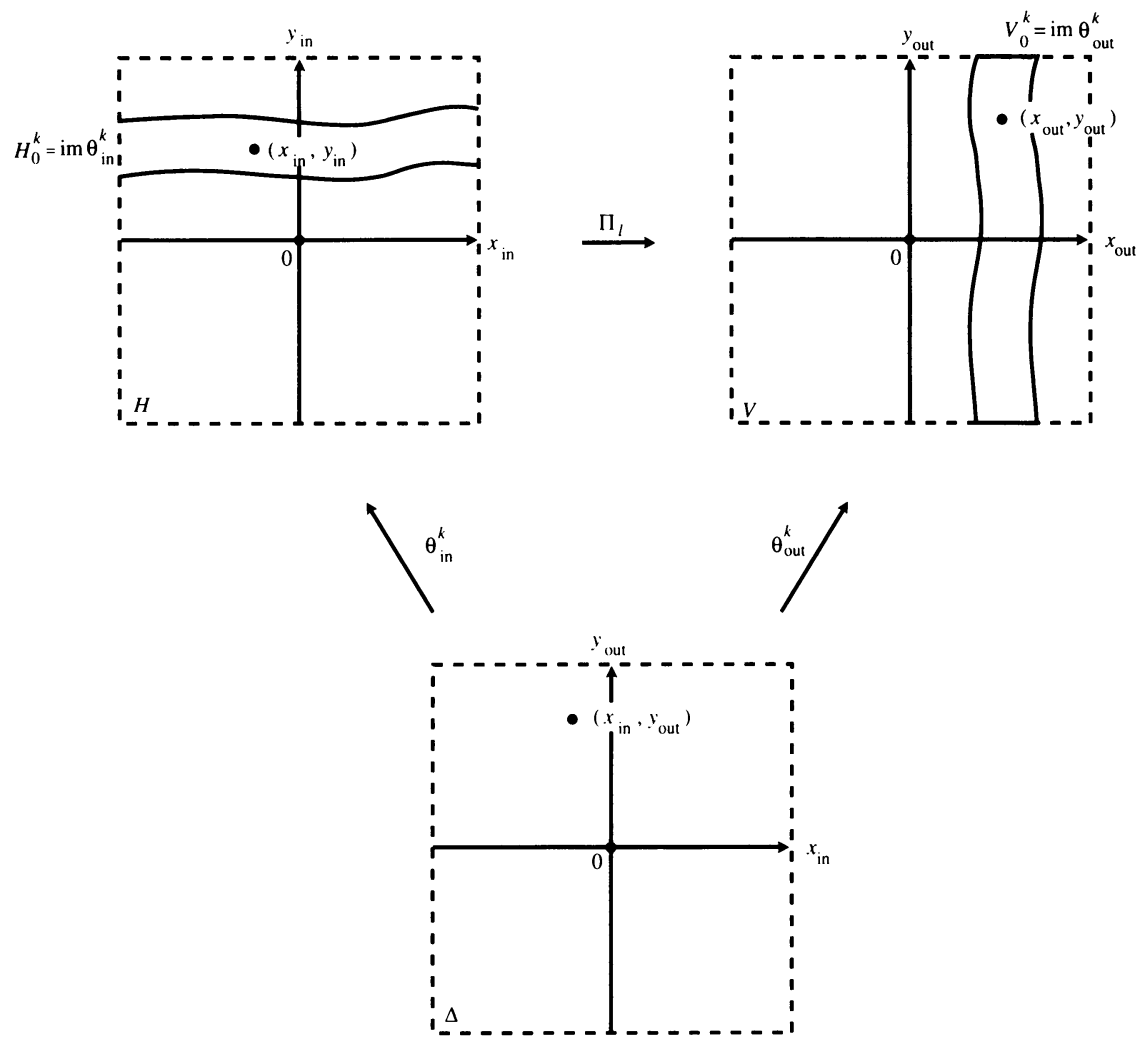

FIGURE 2.2 
led to the following system of infinite equations in the Sil'nikov variables:

$$
\begin{aligned}
& x_{\text {in }}^{j+1}=\Pi_{g}^{x}\left(\hat{x}\left(\xi^{j}\right), \widehat{\Pi}_{g}^{y}\left(\hat{x}\left(\xi^{j}\right), \hat{y}\left(\xi^{j+1}\right)\right)\right), \\
& y_{\text {out }}^{j+1}=\widehat{\Pi}_{g}^{y}\left(\hat{x}\left(\xi^{j+1}\right), \hat{y}\left(\xi^{j+2}\right)\right), \quad \text { with } \xi^{i}=\left(s_{i}, x_{\text {in }}^{i}, y_{\text {out }}^{i}\right) .
\end{aligned}
$$

Observe that, if we think of the right-hand side as a map $\Phi_{s}: \Delta^{\mathbf{Z}} \rightarrow \Delta^{\mathbf{Z}}$ parametrized by the doubly infinite sequence $s$ of integers $\in N$, then the doubly infinite sequence of the Sil'nikov variables $\zeta=\left(\cdots\left(x_{\text {in }}^{j}, y_{\text {out }}^{j}\right),\left(x_{\text {in }}^{j+1}, y_{\text {out }}^{j+1}\right) \cdots\right)$ on the left-hand side must be a fixed point of $\Phi_{s}$. Here, $\Delta^{\mathbf{Z}}$ denotes the doubly product space of $\Delta$ and it is complete and metrizable with, e.g., the metric

$$
d\left(\zeta, \zeta^{\prime}\right)=\sum_{j=-\infty}^{\infty} \frac{1}{2|j|}\left(\left|x_{\text {in }}^{j}-x_{\text {in }}^{j}\right|+\left|y_{\text {out }}^{j}-y_{\text {out }}^{\prime j}\right|\right) .
$$

The infinite sum here is understood as the limit of $\sum_{j=m}^{n}$ as $m \rightarrow-\infty$ and $n \rightarrow+\infty$ independently. Also notice that, under this product topology, $\boldsymbol{\Phi}_{s}$ is a uniform contraction mapping for $s \in N^{\mathbf{Z}}$ if each component $\Phi_{s}^{j}$ is a contraction mapping having a fairly small contraction constant, say less than $\frac{1}{8}$. If this is the case, there will be a unique fixed point $\zeta^{*}(s)$ for every $s \in N^{\mathbf{Z}}$ and it depends continuously on $s$ by the uniform contraction mapping principle. Indeed, the last statement follows from the fact that, by the definition of $\boldsymbol{\Phi}_{s}$ and the metric $d$ on $\Delta^{\mathrm{Z}}$, the function $s \rightarrow \Phi_{s}$ actually is continuous from the product space $N^{\mathbf{Z}}$ into the space of continuous functions in $\Delta^{\mathbf{Z}}$. Thus, we have

Lemma 2.1. Under the above setting, let

$$
\begin{aligned}
& g=g(\delta):=\max _{|z| \leq \delta}\left\{|D F(z)|^{M+1},\left|D F^{-1}(z)\right|^{M+1}\right\}, \\
& L=L\left(\delta, \delta_{0}, \delta_{1}, N_{0}\right)=\sup \{\operatorname{Lip} \hat{x}(k, \cdot, \cdot), \operatorname{Lip} \hat{y}(k, \cdot, \cdot)\} \\
& \text { with }\left|x_{\text {in }}\right| \leq \delta_{0},\left|y_{\text {out }}\right| \leq \delta_{1}, k \in N_{0},
\end{aligned}
$$

where $N_{0} \subset N$ is a subset of $N$ defined as in (2.4a) and Lip denotes the Lipschitz constant of a given function. Suppose the condition (2.5b) and

$$
g L<\frac{1}{8}
$$

are satisfied. Then, for every $s \in N_{0}^{\mathbf{Z}}$, the map $\Phi_{s}$ defined by (2.6) has a unique fixed point $\zeta^{*}=\zeta^{*}(s)$ which is one-to-one. Moreover, let $\zeta^{* j}=\left(x_{\text {in }}^{* j}, y_{\text {out }}^{* j}\right)$ be the $j$ th component of $\zeta^{*}$ and $\theta_{\mathrm{in}}^{s_{0}}$ be the Sil'nikov change of variables defined by $(2.5 \mathrm{c})$ for the initial points in particular. Then the map

$$
\phi:=\theta_{\text {in }}^{s_{0}} \circ \zeta^{* 0}, \quad s \mapsto\left(x_{\text {in }}^{* 0}(s), \hat{y}\left(s_{0}, x_{\text {in }}^{* 0}(s), y_{\text {out }}^{* 0}(s)\right)\right),
$$

defines a topological conjugacy from the shift dynamics $\left\{N_{0}^{\mathbf{Z}}, \sigma\right\}$ onto $\{\operatorname{im} \phi, \Pi\}$, the dynamics of the Poincare map on the image im $\phi$.

Proof. The first half of the lemma has been proved in the discussion preceding the lemma. Indeed, the contraction constant of $\Phi_{s}^{j}$ is at most $g L<\frac{1}{8}$ and 
the space $\Delta^{\mathbf{Z}}$ is a complete metric space. To complete the proof, we only need to show $\phi$ is a homeomorphism onto its image $\operatorname{im} \phi$ which is endowed with the Euclidean topology. That $\phi$ is one-to-one is trivial by the one-to-one correspondence between $\zeta^{*}(s)$ and its associated orbit in $\Omega$, plus the trivial one-to-one correspondence of the orbit with its initial point. It is also continuous by the uniform contraction mapping principle. To show the continuity of the inverse, it suffices to show $\phi$ is an open mapping. But this is due to the following

$$
\phi\left(B_{s_{-l} \cdots s_{0} \cdots s_{k}}\right)=\bigcap_{j=-l}^{k} \Pi^{-j}\left(H_{0}^{s_{j}}\right) \cap \operatorname{im} \phi,
$$

where $B_{s_{-l} \cdots s_{0} \cdots s_{k}}$ is the topological basis element of $s=\left(\cdots s_{-l} \cdots s_{0} \cdots s_{k} \cdots\right) \in$ $N_{0}^{\mathbf{Z}}$ which consists of those points $s^{\prime}$ in $N_{0}^{\mathbf{Z}}$ so that $s_{j}^{\prime}=s_{j}$ for $-l \leq j \leq k$. In fact, by definition, $\phi\left(B_{s_{-l}} \cdots s_{0} \cdots s_{k}\right)$ consists of those initial points whose $j$ th iterate is $\theta_{\text {in }}^{s_{j}} \circ \zeta^{* j}=\left(x_{\text {in }}^{* j}(s), \hat{y}\left(s_{j}, x_{\text {in }}^{* j}(s), y_{\text {out }}^{* j}(s)\right)\right) \in H_{0}^{s_{j}}$ for $-l \leq j \leq k$. Thus, $\phi\left(B_{s_{-l} \cdots s_{0} \cdots s_{k}}\right) \subset \bigcap_{-l \leq j \leq k} \Pi^{-j}\left(H_{0}^{s_{j}}\right) \cap \operatorname{im} \phi$. On the other hand, it is trivial to see $\phi^{-1}\left(\bigcap_{-l \leq j \leq k} \Pi^{-j}\left(H_{0}^{s_{j}}\right) \cap \operatorname{im} \phi\right) \subset B_{s_{-l} \cdots s_{0} \cdots s_{k}}$ by definition. This proves the identity. Moreover, being connected components, $H_{0}^{k}$ are open in $H_{0}$. Hence, the right-hand side set is indeed open in $\operatorname{im} \phi$. This completes the proof.

Next, we introduce another technical lemma which is concerned with forward and backward invariant sets for the Poincare map $\Pi$ on $H$, including those sets $\Omega^{+}, \Omega^{-}$, and $\Omega^{0}$. To begin with, let us recall $\Omega^{+}$from $(1.2 \mathrm{a})$ which is $\bigcup_{n \geq 0}\left\{\Pi^{-n}\left(W_{H}^{\text {cs }}\right) \cap \bigcap_{k \leq n-1} \Pi^{-k}\left(H_{0}\right)\right\}$ in general. Given $z^{0} \in \Omega^{+}$, there is a unique $n \geq 0$ such that $\Pi^{n}\left(z^{0}\right) \in W_{H}^{\text {cs }}$. Thus, there is a unique leftward infinite sequence $s=\left(\cdots s_{-1} s_{0} \cdots s_{n-1}\right)$ such that $\Pi^{j}\left(z^{0}\right) \in H_{0}^{s_{j}}$ for $-\infty<j \leq$ $n-1$. Similarly, if we let $\Pi^{j}\left(z^{0}\right)=\left(x_{\text {in }}^{j}, y_{\text {in }}^{j}\right),\left(x_{\text {out }}^{j}, y_{\text {out }}^{j}\right)=\Pi_{l}\left(x_{\text {in }}^{j}, y_{\text {in }}^{j}\right)$, for $j \leq n-1$, and $\left|x_{\text {in }}^{n}\right| \leq \delta_{0}$ while $y_{\text {in }}^{n}=0$ for $\Pi^{n}\left(z^{0}\right) \in W_{H}^{\text {cs }}=\left\{y_{\text {in }}=0\right\}$ locally, we wind up with the following system of equations with $\beta=y_{\text {in }}^{n}=0$ by (2.3) and (2.5b):

$$
\begin{aligned}
x_{\text {in }}^{n-1} & =\Pi_{g}^{x}\left(\hat{x}\left(\xi^{n-2}\right), \widehat{\Pi}_{g}^{y}\left(\hat{x}\left(\xi^{n-2}\right), \hat{y}\left(\xi^{n-1}\right)\right)\right), \\
y_{\text {out }}^{n-1} & =\widehat{\Pi}_{g}^{y}\left(\hat{x}\left(\xi^{n-1}\right), \beta\right),
\end{aligned}
$$

Notice that the discussion above is also valid for all nonzero $y_{\text {in }}^{n}$ which is treated as a new parameter $\beta$ in (2.8) and it corresponds to the case where $z^{0} \in$ $\Pi^{-n}(H) \cap \bigcap_{k \leq n-1} \Pi^{-k}\left(H_{0}^{s_{k}}\right)$, namely, with $W_{H}^{\text {cs }}$ replaced by $H$, is a point of backward invariance in general. See Figure 2.3. Treating the right-hand side as a map $\Phi_{s, \beta}^{+}$from the product space $\Pi_{k<n} \Delta$ into itself implies that the leftward Sil'nikov sequence $\left(\cdots\left(x_{\text {in }}^{n-2}, y_{\text {out }}^{n-2}\right),\left(x_{\text {in }}^{n-1}, y_{\text {out }}^{n-1}\right)\right)$ must be its fixed point. Notice that the definition of $\Phi_{s, \beta}^{+}$depends on the integer $n$ which is suppressed. Since we will present our lemma in a compact form, let us not rush to state at this moment the obvious conclusion about $\Phi_{s, \beta}^{+}$. 


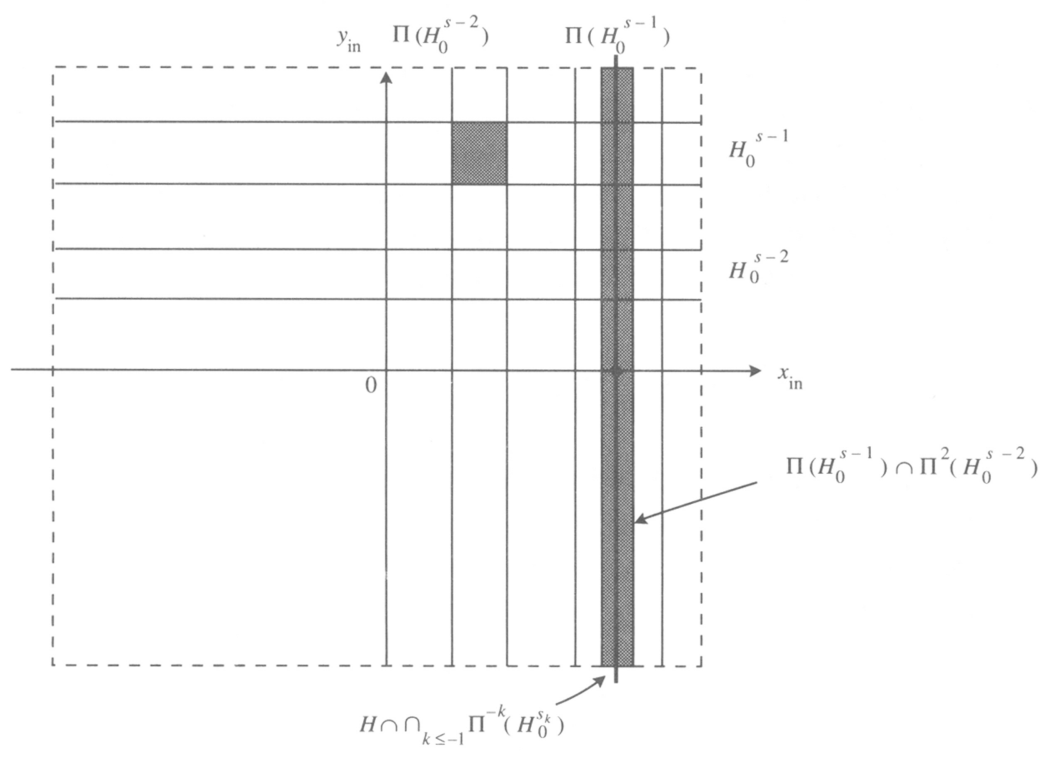

FIGURE 2.3

To continue, let us recall $\Omega^{-}$from $(1.2 b)$ :

$$
\bigcup_{m \leq 0}\left\{\Pi^{-m}\left(W_{H}^{\mathrm{u}}\right) \cap \bigcap_{k \geq m+1} \Pi^{-k}\left(H_{0}\right)\right\} .
$$

Following the same argument, we have that given a $\left(x_{\text {in }}^{0}, y_{\text {in }}^{0}\right) \in \Omega^{-}$there is a unique $m \leq 0$ and a sequence $s=\left(s_{m} \cdots s_{0} s_{1} \cdots\right)$ such that $\left(x_{\text {in }}^{j}, y_{\text {in }}^{j}\right)=$ $\Pi^{j}\left(x_{\text {in }}^{0}, y_{\text {in }}^{0}\right) \in H_{0}^{s_{j}}$ for $m \leq j$, and in particular, $\left(x_{\text {in }}^{m}, y_{\text {in }}^{m}\right) \in W_{H}^{\mathrm{u}} \cap H_{0}^{s_{m}}$. Thus, $\left(x_{\text {out }}^{m-1}, y_{\text {out }}^{m-1}\right)=\left(0, y_{\text {out }}^{m-1}\right) \in W_{\text {loc }}^{\mathrm{u}} \cap B\left(p_{1}, \delta_{1}\right)$, and $y_{\text {out }}^{m-1}=\widehat{\Pi}_{g}^{y}\left(x_{\text {out }}^{m-1}, y_{\text {in }}^{m}\right)$ with $x_{\text {out }}^{m-1}=0$. Also, the discussion above is valid for all nonzero $x_{\text {out }}^{m-1}$ which correspond to the case where $\left(x_{\text {in }}^{0}, y_{\text {in }}^{0}\right) \in \Pi^{-m}(H) \cap \bigcap_{k>m+1} \Pi^{-k}\left(H_{0}^{s_{k}}\right)$. Therefore, such general points $\left(x_{\text {in }}^{0}, y_{\text {in }}^{0}\right)$ of forward invariance are associated with the following system of infinite equations of the Sil'nikov variables $\zeta=$ $\left(\left(x_{\text {in }}^{m}, y_{\text {out }}^{m}\right),\left(x_{\text {in }}^{m+1}, y_{\text {out }}^{m+1}\right), \ldots\right)$ parametrized by the sequences together with the parameter $\beta:=x_{\text {out }}^{m-1}$ :

$$
\begin{aligned}
x_{\text {in }}^{m} & =\Pi_{g}^{x}\left(\beta, \widehat{\Pi}_{g}^{y}\left(\beta, \hat{y}\left(\xi^{m}\right)\right)\right), \\
x_{\text {out }}^{m} & =\widehat{\Pi}_{g}^{y}\left(\hat{x}\left(\xi^{m}\right), \hat{y}\left(\xi^{m+1}\right)\right), \quad \text { with } \xi^{i}=\left(s_{i}, x_{\text {in }}^{i}, y_{\text {out }}^{i}\right) .
\end{aligned}
$$

Again, we have derived a map, called $\Phi_{s, \beta}^{-}$on the one-side infinite product of the Sil'nikov space $\prod_{m \leq k} \Delta$, whose fixed points correspond to the set $\Pi^{-m}(H) \cap$ $\bigcap_{k \geq m+1} \Pi^{-k}\left(H_{0}^{s_{k}}\right)$. The dependence of $\Phi_{s, \beta}^{-}$on $m$ is also suppressed. Last, combining the two arguments above, we conclude that for fixed $m \leq 0 \leq n$, the subset $\left\{\Pi^{-m}(H) \cap \bigcap_{k=m+1}^{n-1} \Pi^{-k}\left(H_{0}\right) \cap \Pi^{-n}\left(W_{H}^{c s}\right)\right\}$, intersecting $\Omega^{0}$, is in one-to-one correspondence with the fixed points of a map $\Phi_{s, \beta}^{0}$ in $\prod_{m \leq k<n} \Delta$ for all possible $s=\left(s_{m} \cdots s_{0} \cdots s_{n-1}\right)$ and $|\beta| \leq \delta_{1}$. Here, $\Phi_{s, \beta}^{0}$ is defined as 
the right-hand side of the following equations:

$$
\begin{aligned}
x_{\text {in }}^{m}= & \Pi_{g}^{x}\left(\beta, \widehat{\Pi}_{g}^{y}\left(\beta, \hat{y}\left(\xi^{m}\right)\right)\right), \\
y_{\text {out }}^{m}= & \widehat{\Pi}_{g}^{y}\left(\hat{x}\left(\xi^{m}\right), \hat{y}\left(\xi^{m+1}\right)\right), \quad \text { with } \xi^{i}=\left(s_{i}, x_{\text {in }}^{i}, y_{\text {out }}^{i}\right) . \\
\vdots & \\
y_{\text {out }}^{n}= & \widehat{\Pi}_{g}^{y}\left(\hat{x}\left(\xi^{n-1}\right), 0\right),
\end{aligned}
$$

Notice that when $m=0=n$, it trivially corresponds to the homoclinic point $p_{0}$. We also note that the operators $\Phi_{s, \beta}^{i}$ are continuous in $s$ and $\beta$. We now have

Lemma 2.2. Assume the same conditions (2.5b) and (2.7) of Lemma 2.1. Let $N_{0}, N$ be the same as in that lemma. Then

(a) for every $n \geq 0$, the product space $N_{0}^{\{\cdots n-2, n-1\}} \times\left\{|\beta| \leq \delta_{0}\right\}$ is homeomorphic to $\Pi^{-n}(H) \cap \bigcap_{k \leq n-1} \Pi^{-k}\left(\bigcup_{j \in N_{0}} H_{0}^{j}\right)$. Specifically, let $\zeta^{+}(s, \beta)$ be the unique fixed point of $\Phi_{s, \beta}^{+}$defined by $(2.8)$; then $\phi^{+}:=\theta_{\text {in }}^{s_{0}} \circ \zeta^{+, 0}$ defines the homeomorphism.

(b) Similar statements hold true for equations (2.9) and (2.10) respectively. Specifically, for every $m \leq 0 \leq n$, the product $N_{0}^{\{m, m+1, \ldots\}} \times\left\{|\beta| \leq \delta_{1}\right\}$ $\left(N_{0}^{\{m, m+1, \ldots, n-1\}} \times\left\{|\beta| \leq \delta_{1}\right\}\right.$ resp. $)$ is homeomorphic to

$$
\begin{aligned}
\Pi^{-m}(H) \cap \bigcap_{k \geq m+1} \Pi^{-k} & \left(\bigcup_{j \in N_{0}} H_{0}^{j}\right) \\
& \left(\Pi^{-m}(H) \cap \bigcap_{k \geq m+1}^{n-1} \Pi^{-k}\left(\bigcup_{j \in N_{0}} H_{0}^{j}\right) \cap \Pi^{-n}\left(W_{H}^{\mathrm{cs}}\right) \text { resp. }\right) .
\end{aligned}
$$

Then the homeomorphism is defined as $\phi^{-}:=\theta_{\text {in }}^{s_{0}} \circ \zeta^{-, 0} \quad\left(\phi^{0}:=\theta_{\text {in }}^{s_{0}} \circ \zeta^{0,0}\right.$ resp. $)$, where $\zeta^{i}(s, \beta)$ denotes the fixed point of $\Phi_{s, \beta}^{i}$ defined by (2.9) and (2.10).

(c) Moreover, if the Sil'nikov changes of variables (2.5c) and the maps $\Pi_{g}^{x}, \widehat{\Pi}_{g}^{y}$ are $C^{l}$, then the functions $\zeta^{i}(s, \beta)$ are also $C^{l}$ in $\beta$ for $i \in\{+,-, 0\}$. Furthermore, for every sequence $s=\left(\cdots s_{n-2} s_{n-1}\right)$ (or $s=\left(s_{m} s_{m+1} \cdots\right)$, or segment $s=\left(s_{m} \cdots s_{n-1}\right)$ resp.) with $s_{j} \in N_{0}$, the set $\Pi^{-n}(H) \cap \bigcap_{k \leq n-1} \Pi^{-k}\left(H_{0}^{s_{k}}\right)$ (or $\Pi^{-m}(H) \cap \bigcap_{k \geq m+1} \Pi^{-k}\left(H_{0}^{s_{k}}\right)$, or $\Pi^{-m}(H) \cap \bigcap_{k \geq m+1}^{n-1} \Pi^{-k}\left(H_{0}^{s_{k}}\right) \cap \Pi^{-n}\left(W_{H}^{c s}\right)$ resp. $)$ is diffeomorphic to the $C^{l}$ vertical (or horizontal resp.) graph

$$
\Pi_{g}^{x}\left(\hat{x}\left(s_{n-1}, x_{\text {in }}^{+, n-1}(s, \cdot), y_{\text {out }}^{+, n-1}(s, \cdot)\right), y_{\text {out }}^{+, n-1}(s, \cdot)\right) \text { in } H
$$

(or

or

$$
\widehat{\Pi}_{g}^{y}\left(\cdot, \hat{y}\left(s_{m}, x_{\text {in }}^{-}, m(s, \cdot)\right), y_{\text {out }}^{-, m}(s, \cdot)\right) \text {, }
$$

resp. in $V$ ).

$$
\widehat{\Pi}_{g}^{y}\left(\cdot, \hat{y}\left(s_{m}, x_{\text {in }}^{0, m}(s, \cdot)\right), y_{\text {out }}^{0, m}(s, \cdot)\right)
$$

Proof. The proof is a straightforward application of the uniform contraction mapping principle to the maps $\Phi_{s, \beta}^{i}, i \in\{+,-, 0\}$, and of the same argument as in the proof of Lemma 2.1 for the homeomorphic property. 
Recall the definitions of the sets of doubly infinite sequences $S, S^{+}, S^{-}$, and $S^{0}$ from (1.3) which are incorporated with the symbols $\infty^{\mathrm{s}}$ and $\infty^{\mathrm{u}}$. Replace the symbols $s_{i} \geq K_{0}$ by $s_{i} \in N_{0}$, with $N_{0}$ the same as in the lemmas above, and denote the newly defined sets as $S\left(N_{0}\right), S^{i}\left(N_{0}\right), i \in\{+,-, 0\}$. Also, let $\widehat{S}\left(N_{0}\right)$ denote the corresponding standard blowups and equip the union of blowups

$$
\widehat{\mathscr{S}}\left(N_{0}\right):=\widehat{S}\left(N_{0}\right) \cup\left\{\widehat{S}^{i}\left(N_{0}\right), i \in\{+,-, 0\}\right\}
$$

with the topology generated by that of its components. Then, an immediate conclusion from Lemmas 2.1 and 2.2 above is that one can define a one-toone and onto map $\psi: \widehat{\mathscr{S}} \rightarrow \mathscr{A}$ as $\psi(\hat{s}):=\phi(s)$, or $\phi^{i}(s, 0)$ accordingly for $i \in\{+,-, 0\}$, where

$$
\mathscr{A}:=\Omega\left(N_{0}\right) \cup\left\{\Omega^{i}\left(N_{0}\right): i \in\{+,-, 0\}\right\}
$$

and $s$ and $\hat{s}$ are the standard sequences and the corresponding standard blowup sequences respectively. Here, obviously in the same logic, $\Omega\left(N_{0}\right)$ and $\Omega^{i}\left(N_{0}\right)$ are the same as $\Omega$ and $\Omega^{i}$ defined in $(1.2 \mathrm{a})-(1.2 \mathrm{c})$ except that $H_{0}$ here is replaced by $\bigcup_{k \in N_{0}} H_{0}^{k}$. It is also understood that the augmented and doubly infinite sequences have a natural correspondence to their original and one-sided infinite sequences. Thus, we did not make the distinction among them when we wrote $\phi^{i}(s, 0)$ above. The one-to-one property of $\psi$ follows from the fact that not only are $\Omega$ and $\Omega^{i}$ distinct (i.e., mutually having empty intersection) but also the components in the union of each $\Omega^{i}$ are disjoint by the remark preceding Theorem 1.2. Recall that this is simply due to the iterative disjointness of the chosen sets $H$ and $V$ from (2.1a). As the last result of this section, we have

Lemma 2.3. Under the setting above, the inverse of $\psi$ is continuous from $\mathscr{A}$ onto $\widehat{\mathscr{S}}$.

Proof. To show the continuity of $\psi^{-1}$ it suffices to show $\psi$ is an open mapping. To do so, let $B_{\hat{s}_{-} \cdots \hat{s}_{0} \cdots \hat{s}_{k}}$ be a typical topological basis element of $\widehat{\mathscr{S}}$ which consists of those points $\hat{s}^{\prime}$ such that for all $-l \leq i \leq k$ either $s_{i}^{\prime}=s_{i}$ or $b_{i} \leq s_{i}^{\prime}<\infty$ only if $s_{i}^{\prime} \neq s_{i} \in\left\{\infty^{\mathrm{s}}, \infty^{\mathrm{u}}\right\}$, where the $b_{i}$ are some constants to be specified as follows. If we let $n$ and $m-1$ be the least and largest number such that $s_{n}=\infty^{\mathrm{s}}$ and $s_{m-1}=\infty^{\mathrm{u}}$ respectively, then the basis element is so chosen that $b_{i} \geq \max \{|k-n|,|l+m|\}$. We claim that the openness of $\psi$ follows from the identity

$$
\begin{aligned}
\psi\left(B_{\hat{s}_{-l} \cdots \hat{s}_{0} \cdots \cdots \hat{s}_{k}}\right)= & \left\{\bigcup H_{0}^{j} \cup \Pi^{-m+1}\left(\bigcup_{j>b_{n}} H_{0}^{j}\right)\right\} \\
& \cap \bigcap_{m \leq i<n} \Pi^{-i}\left(H_{0}^{s_{i}}\right) \cap \Pi^{-n}\left(W_{H}^{\mathrm{cs}} \bigcup_{j \geq b_{n}} H_{0}^{j}\right) \cap \operatorname{im} \psi,
\end{aligned}
$$

where, of course, all $s_{i}$ and $j$ are from $N_{0}$. Indeed, for every given element $\hat{s}^{\prime}$ from the open set either $s_{i}^{\prime}=s_{i}$ or the blowup $\hat{s}_{n}^{\prime}$ covers the entire segment from the $n$th place to the $k$ th one prior to blowing up because of the choice of $b_{n}$. Similarly, if $s_{m-1}^{\prime} \neq \infty^{\mathrm{u}}$ then $\hat{s}_{m-1}^{\prime}$ covers the segment from the 'lth' place to the ' $(m-1)$ st' one. This implies $\Pi^{i} \circ \psi\left(\hat{s}^{\prime}\right) \in H_{0}^{s_{i}}$ for all $m \leq i<n$, 
$\Pi^{m-1} \circ \psi\left(\hat{s}^{\prime}\right) \in H_{0}^{s_{m-1}^{\prime}}$ if $b_{m-1} \leq s_{m-1}^{\prime} \neq \infty^{\mathrm{u}}$, and $\Pi^{n} \circ \psi\left(\hat{s}^{\prime}\right) \in W_{H}^{\mathrm{cs}}$ if $s_{n}^{\prime}=\infty^{\mathrm{s}}$ or $H_{0}^{s_{n}^{\prime}}$ if $b_{n} \leq s_{n}^{\prime} \neq \infty^{\mathrm{s}}$. Conversely, it is also clear to see that if $z^{\prime} \in \operatorname{im} \psi$ satisfies the properties above, then its associated blowup $\hat{s}^{\prime}$ must be in the given basis element. This proves the identity. To see the openness of the subset of $\operatorname{im} \psi$ on the right-hand side of the identity, observe that since the $H_{0}^{j}$ 's are connected components, they are both open and closed in $\cup H_{0}^{j} \cup W_{H}^{\text {cs }}$, and $W_{H}^{\text {cs }} \cup \bigcup_{j \geq b_{n}} H_{0}^{j}$ is open since its complement is the finite union of the closed sets $H_{0}^{j}$ with $j \leq b_{n}$. Here, $j \in N_{0}$. Since $\Pi$ is a homeomorphism, the subset is indeed open in $\operatorname{im} \psi$.

\section{Proofs of Theorems $1.1-1.6$}

The key step in proving Theorems 1.1 and 1.2 is to check condition (2.5b). This in turn is a simple consequence to the following result whose proof can be found in Deng (1988a,b).

Proposition 3.1. Let $(x, y)=0$ be a hyperbolic fixed point of $F \in C^{r}, r \geq 1$, and the coordinate $(x, y)$ be normalized in such a way that $W_{\mathrm{loc}}^{\mathrm{u}}=\{x=0\}$ and $W_{\mathrm{loc}}^{\mathrm{s}}=\{y=0\}$ locally. Then there exists $a \delta>0$ such that for every triplet $\left(l, x_{0}, y_{1}\right)$, with $l \geq 0$ any integer and $\left|x_{0}\right|,\left|y_{1}\right| \leq \delta$, there is a unique local orbit $\gamma=\left\{\left(x^{i}, y^{i}\right): 0 \leq i \leq l\right\}$ contained in the $2 \delta$-box $B(2 \delta)$ satisfying

$$
x^{0}=x_{0} \text { and } y^{l}=y_{1} .
$$

Denote this correspondence as $\left(x^{i}, y^{i}\right)\left(l, x_{0}, y_{1}\right)$. Then $x^{l}(l, \cdot, \cdot)$ and $y^{0}(l, \cdot, \cdot)$ are $C^{r}$ functions which converge in the $C^{r}$ uniform norm to $x=0$ and $y=0$ respectively as $l \rightarrow+\infty$.

A heuristic illustration of this result is shown in Figure 3.1.

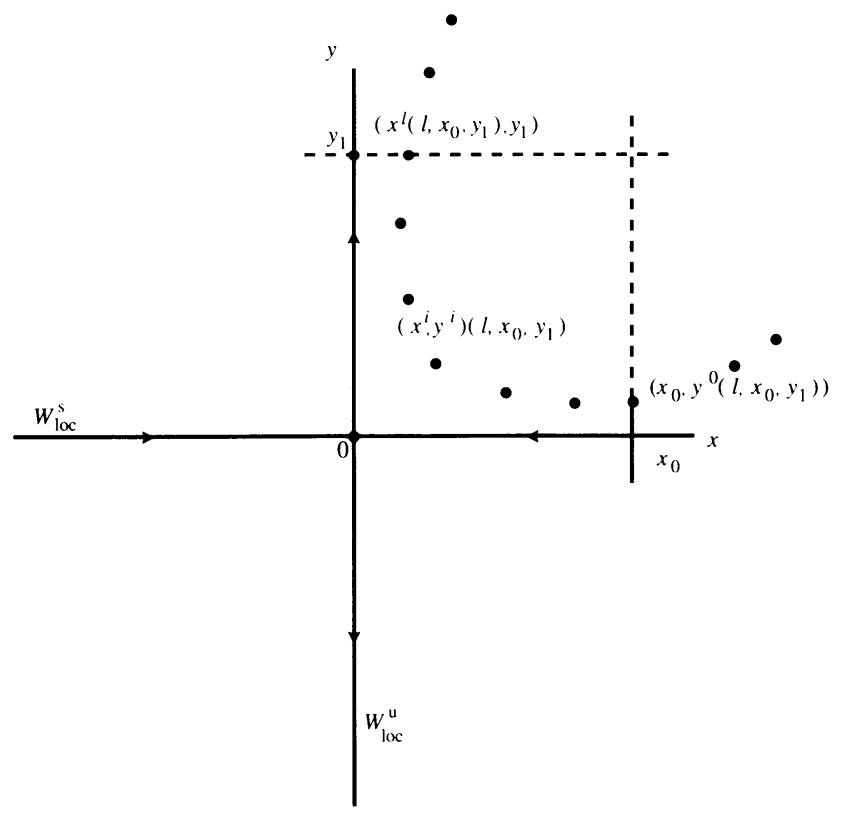

FIGURE 3.1 
Proof of Theorem 1.1. To check the condition (2.5b), let $k=l+M+1$ and define

(3.1a) $\hat{x}\left(k, x_{\text {in }}, y_{\text {out }}\right):=x^{l}\left(l, x_{0}, y_{1}\right), \quad \hat{y}\left(k, x_{\text {in }}, y_{\text {out }}\right):=y^{0}\left(l, x_{0}, y_{1}\right)$,

where $\left(x_{\mathrm{in}}, 0\right)$ is the local coordinate of $\left(x_{0}, 0\right)$ from the horizontal box $H=B\left(p_{0}, \delta_{0}\right)$ and $\left(0, y_{\text {out }}\right)$ is the local coordinate of $\left(0, y_{1}\right) \in V=B\left(p_{1}, \delta_{1}\right)$. Because of the uniform $C^{r}$ convergence of $x^{l}(l, \cdot, \cdot)$ and $y^{0}(l, \cdot, \cdot)$ by Proposition 3.1 , we can choose and fix a large integer $K_{0}\left(\delta, \delta_{0}, \delta_{1}\right)$ so that for all $k \geq K_{0}$,

$$
\begin{gathered}
\|\hat{x}(k, \cdot, \cdot)\| \leq \delta_{1}, \quad\|\hat{y}(k, \cdot, \cdot)\| \leq \delta_{0}, \quad \text { and } \\
\|D \hat{x}(k, \cdot, \cdot)\|+\|D \hat{y}(k, \cdot, \cdot)\|<\frac{1}{8 g},
\end{gathered}
$$

where the constant $g=\max _{|z| \leq \delta}\left\{|D F(z)|^{M+1},\left|D F^{-1}(z)\right|^{M+1}\right\}$ is the same as in Lemma 2.1. Choose $N_{0}=\left\{K_{0}, K_{0}+1, \ldots\right\}$, then Lemma 2.1 applies. Hence, by choosing $H_{0}=\bigcup_{k \geq K_{0}} H_{0}^{k}$, the two dynamical systems $\left\{N_{0}^{\mathrm{Z}}, \sigma\right\}$ and $\{\Omega, \Pi\}$ are topologically conjugate.

Proof of Theorem 1.2. Choose $\hat{x}, \hat{y}, K_{0}, N_{0}$, and $H_{0}$ the same as in the proof above. It is obvious that $N_{0}^{\{\cdots, n-2, n-1\}}$ is in one-to-one correspondence with $\left\{\Pi^{-n}\left(W_{H}^{\mathrm{s}}\right) \cap \bigcap_{k \leq n-1} \Pi^{-k}\left(H_{0}\right)\right\}$ by Lemma 2.2 . Since these sets are mutually exclusive for distinct $n$, the conclusion of Theorem 1.2 for $\Omega^{+}$follows. Last, fixing the parameter $\beta=x_{\text {out }}^{n-1}=0$, which corresponds to $W_{\mathrm{loc}}^{\mathrm{u}} \cap V$, the other part of the theorem follows immediately.

As one might have noticed, Theorem 1.2 is much weaker than Lemma 2.2. We stated it as it was simply for an accurate count of credits due to Sil'nikov.

Proof of Theorem 1.3. In terms of the Sil'nikov changes of variables $(2.5 \mathrm{c})$, we have the following simple facts:

$$
H_{0}^{k}=\bigcup_{\left|y_{\text {out }}\right| \leq \delta_{1}} \operatorname{graph}\left(\hat{y}\left(k, \cdot, y_{\text {out }}\right)\right) \quad \text { and } \quad V_{0}^{k}=\bigcup_{\left|x_{\text {in }}\right| \leq \delta_{1}} \operatorname{graph}\left(\hat{x}\left(k, x_{\text {in }}, \cdot\right)\right),
$$

where $V_{0}=\Pi_{l}\left(H_{0}\right)$ and $V_{0}^{k}=\Pi_{l}\left(H_{0}^{k}\right)$; and thus $W_{H}^{\mathrm{s}}=W_{\mathrm{loc}}^{\mathrm{s}} \cap H$ and $W_{V}^{\mathrm{u}}=$ $W_{\text {loc }}^{\mathrm{u}} \cap V$ are the only limiting sets for $\left\{H_{0}^{k}\right\}_{k \geq K_{0}}$ and $\left\{V_{0}^{k}\right\}_{k \geq K_{0}}$ respectively by the uniform convergence of Proposition 3.1 (cf. Figure 1.3). Namely

$$
\bar{H}_{0}=H_{0} \cup W_{H}^{\mathrm{s}} \text { and } \bar{V}_{0}=V_{0} \cup W_{V}^{\mathrm{u}} \text {. }
$$

Let $\widetilde{\Omega}:=\Omega \cup \Omega^{+} \cup \Omega^{-} \cup \Omega^{0}$. To prove the first half of the theorem we must show $\bar{\Omega}=\widetilde{\Omega}$. We begin by showing $\widetilde{\Omega} \subset \bar{\Omega}$ which in turn is equivalent to showing $\Omega^{i} \subset \bar{\Omega}$ for $i \in\{+,-, 0\}$. We start the case with $i=+$.

For every given $z^{0} \in \Omega^{+}$we may assume, up to some finite iterate of $\Pi$, that $z^{0} \in W_{H}^{\text {cs }}$ as well. Then by Lemma 2.2 there is a leftward sequence $s=$ $\left(\cdots s_{-2} s_{-1}\right)$ such that $z^{0} \in W_{H}^{\text {cs }} \cap \bigcap_{k<-1} \Pi^{-k}\left(H_{0}^{s_{k}}\right)$. Also by that lemma $z^{0}$ is in the vertical graph $\mathscr{G}^{+}(s, \cdot)$ of backward invariance in $H$ parametrized by $s$, where

$$
\mathscr{G}^{+}(s, \beta):=\Pi_{g}^{x}\left(\hat{x}\left(s_{-1}, x_{\text {in }}^{+,-1}(s, \beta), y_{\text {out }}^{+,-1}(s, \beta)\right), y_{\text {out }}^{+,-1}(s, \beta)\right) .
$$

In particular, $z^{0}=\left(\mathscr{G}^{+}(s, 0), 0\right)$ (cf. Figure 2.3). Let $z_{k}$ be the point from $\Omega$ corresponding to the rightward augmented and doubly infinite sequence 
$\left(\cdots s_{-2} s_{-1} k \cdots k \cdots\right)$. Then $z_{k}$ must tend to $z^{0}$ as $k \rightarrow \infty$. Indeed, sharing the same leftward sequences implies that $z_{k}:=\left(x_{\mathrm{in}, k} y_{\mathrm{in}, k}\right)$ also belongs to the graph $\mathscr{G}^{+}$for all $k \geq K_{0}$ by Lemma 2.2, i.e., $x_{\text {in }, k}=\mathscr{G}^{+}\left(s, y_{\text {in }, k}\right)$. But on the other hand, $y_{\text {in }, k}=\hat{y}\left(k, x_{\text {in }, k}, y_{\text {out }, k}\right)$ since $z_{k} \in H_{0}^{k}$. Since $\hat{y}(k, \cdot, \cdot)$ converges to zero as $k \rightarrow \infty$ by Proposition 3.1, this implies $z_{k} \rightarrow\left(\mathscr{G}^{+}(s, 0), 0\right)=$ $z^{0}$ as $k \rightarrow \infty$.

Next, we show $\Omega^{0} \subset \bar{\Omega}$. Actually we proceed to show $\Omega^{0} \subset \bar{\Omega}^{+}$, the closure of $\Omega^{+}$. For every given $z^{0} \in \Omega^{0}$ we may similarly assume, up to some finite iterate of $\Pi$, that $z^{0} \in W_{H}^{u}$. Thus, $z^{\prime}:=\Pi_{g}^{-1}\left(z^{0}\right) \in W_{V}^{u}$. Let $s=\left(s_{0} \cdots s_{n}\right)$ correspond to $z^{0}$ by Lemma 2.2 and $z_{k} \in \Omega^{+}$correspond to the leftward augmented sequence $\left(\cdots k \cdots k s_{0} \cdots s_{n}\right)$. By the same lemma again $z^{\prime}$ and $z_{k}^{\prime}:=\Pi_{g}^{-1}\left(z_{k}\right)$ are in the same horizontal graph $\mathscr{G}^{0}(s, \cdot)$ in $V$, where

$$
\mathscr{G}^{0}(s, \boldsymbol{\beta}):=\widehat{\Pi}_{g}^{y}\left(\boldsymbol{\beta}, \hat{y}\left(s_{0}, x_{\text {in }}^{0,0}(s, \beta), y_{\text {out }}^{0,0}(s, \beta)\right)\right) .
$$

In particular, $z^{\prime}=\left(0, \mathscr{G}^{0}(s, 0)\right)$. By a similar reason as in the case $\Omega^{+} \subset \bar{\Omega}$, we have $z_{k}^{\prime}:=\left(x_{\text {out }, k}^{\prime}, y_{\text {out }, k}^{\prime}\right) \in V_{0}^{k}$ and $x_{\text {out }, k}^{\prime}=\hat{x}\left(k, x_{\text {in }, k}^{\prime}, y_{\text {out }, k}^{\prime}\right) \rightarrow 0$ uniformly as $k \rightarrow \infty$ by Proposition 3.1. Now, the limit $z_{k}^{\prime} \rightarrow z^{\prime}$ follows from

$$
\left(x_{\text {out }, k}^{\prime}, \mathscr{G}^{0}\left(s, x_{\text {out }, k}^{\prime}\right)\right) \rightarrow\left(0, \mathscr{G}^{0}(s, 0)\right) \text { as } k \rightarrow \infty .
$$

It is not hard to see now that $\Omega^{-} \subset \bar{\Omega}$. Indeed, replacing the segment above by a rightward sequence $\left(s_{0} s_{1} \cdots\right)$ corresponding to a given point from $\Omega^{-}$, and replacing $z_{k}$ by that from $\Omega$ corresponding to $\left(\cdots k \cdots k s_{0} s_{1} \cdots\right)$, the same reasoning as in the case $\Omega^{0} \subset \bar{\Omega}$ above falls through. This shows $\tilde{\Omega} \subset \bar{\Omega}$.

To show $\bar{\Omega} \subset \widetilde{\Omega}$ we need the following claim:

$$
\begin{aligned}
& \text { If } z^{0} \notin \Omega \cup \Omega^{+} \cup \Omega^{-} \cup \Omega^{0} \text {, then there are } m \leq 0 \leq n \text { such that } \\
& \text { either } \\
& \qquad \Pi^{n}\left(z^{0}\right) \in H-H_{0} \cup W_{H}^{\mathrm{s}}
\end{aligned}
$$

or

$$
\Pi^{m}\left(z^{0}\right) \in H-\Pi_{g}\left(V_{0} \cup W_{V}^{u}\right) .
$$

To prove the claim, we need to be more precise. Indeed, by assumption, $z^{0}$ is none of the following:

(i) $\Pi^{k}\left(z^{0}\right) \in H_{0}$ are defined for all $k \in \mathbf{Z}$,

(ii) there is an $n \geq 0$ such that $\Pi^{k}\left(z^{0}\right) \in H_{0}$ are defined for all $k \leq n-1$ and $\Pi^{n}\left(z^{0}\right) \in W_{H}^{\mathrm{s}}$,

(iii) there is an $m \leq 0$ such that $\Pi^{k}\left(z^{0}\right) \in H_{0}$ are defined for all $k \geq m+1$ and $\Pi^{m}\left(z^{0}\right) \in W_{H}^{\mathrm{u}}=\Pi_{g}\left(W_{V}^{\mathrm{u}}\right)$,

(iv) there are $m \leq 0 \leq n$ such that $\Pi^{k}\left(z^{0}\right) \in H_{0}$ are defined for all $m+1 \leq$ $k \leq n-1$ and $\Pi^{m}\left(z^{0}\right) \in W_{H}^{\mathrm{u}}=\Pi_{g}\left(W_{V}^{\mathrm{u}}\right), \Pi^{n}\left(z^{0}\right) \in W_{H}^{\mathrm{s}}$.

But, on the other hand, the negation of (i) implies that $z^{0}$ must satisfy one of the following:

(a) there is an $n \geq 0$ such that $\Pi^{k}\left(z^{0}\right) \in H_{0}$ are defined for all $k \leq n-1$ and $\Pi^{n}\left(z^{0}\right) \notin H_{0}$,

(b) there is an $m \leq 0$ such that $\Pi^{k}\left(z^{0}\right) \in H_{0}$ are defined for all $k \geq m+1$ and $\Pi^{m}\left(z^{0}\right) \notin \Pi_{g}\left(V_{0}\right)$,

(c) there are $m \leq 0 \leq n$ such that $\Pi^{k}\left(z^{0}\right) \in H_{0}$ are defined for all $m+1 \leq$ $k \leq n-1$ and $\Pi^{n}\left(z^{0}\right) \notin H_{0}, \Pi^{m}\left(z^{0}\right) \notin \Pi_{g}\left(V_{0}\right)$. 
Now, the claim easily follows from all the combinations of statements (a)-(c) and (i)-(iv).

We now resume our proof that $\bar{\Omega} \subset \widetilde{\Omega}$ by assuming to the contrary that there are $z^{0} \notin \widetilde{\Omega}$ and $z_{i} \in \Omega$ such that $z_{i} \rightarrow z^{0}$ as $i \rightarrow+\infty$. By the claim, let us assume it is the case that $\Pi^{n}\left(z^{0}\right) \in H-H_{0} \cup W_{H}^{\text {s }}$ for some $n \geq 0$. Then by the closedness of $H_{0} \cup W_{H}^{\text {s }}$ from (3.2) there is a small neighborhood $B\left(\Pi^{n}\left(z^{0}\right), \varepsilon\right) \subset$ $H-H_{0} \cup W_{H}^{\mathrm{s}}$. In limit, $\Pi^{n}\left(z_{i}\right) \in B\left(\Pi^{n}\left(z^{0}\right), \varepsilon\right)$ as $i \rightarrow+\infty$, contradicting $z_{i} \in \Omega$. For the other case, we have $B\left(\Pi^{m}\left(z^{0}\right), \varepsilon\right) \subset H-\Pi_{g}\left(V_{0} \cup W_{V}^{u}\right)$ for a small $\varepsilon>0$. The same contradiction arises.

To complete the proof, we only need to show the identity $\Lambda(F, U)=$ $\bigcup_{n \in \mathbf{Z}} F^{n}(\bar{\Omega}) \cup\{0\}$ since the other equivalent identities follow from it immediately. Here $U$ is chosen as

$$
U=B(\delta) \cup \bigcup_{i=1}^{M} U_{i},
$$

where $U_{i}=F^{i}(V)$ (cf. Figure 1.2). It is obvious that we only need to show

$$
\Lambda(F, U) \subset \bigcup_{n \in \mathbf{Z}} F^{n}(\overline{\mathbf{\Omega}}) \cup\{0\} .
$$

Suppose the contrary and let $z^{0} \in \Lambda(F, U)-\bigcup_{n \in \mathbf{Z}} F^{n}(\bar{\Omega}) \cup\{0\}$. Then, either $z^{0} \in U_{i_{0}}$ for some $i_{0}$ or $z^{0} \in B(\delta)$. Since $z^{0} \neq 0$, up to some finite iterate, it must be in $\bigcup_{i=1}^{M} U_{i}$. Thus, without loss of generality, we assume the first case. Hence, $z^{\prime}:=F^{M+1-i_{0}}\left(z^{0}\right) \in H$. Since $z^{\prime} \notin \bar{\Omega}=\Omega \cup \Omega^{+} \cup \Omega^{-} \cup \Omega^{0}$, by the claim (3.3) there are $m \leq 0 \leq n$ such that either $\Pi^{n}\left(z^{\prime}\right) \in H-H_{0} \cup W_{H}^{\mathrm{s}}$ or $\Pi^{m}\left(z^{\prime}\right) \in H-\Pi_{g}\left(V_{0} \cup W_{V}^{\mathrm{u}}\right)$. Suppose it is the first case; then $\Pi^{n}\left(z^{\prime}\right)$ will eventually exit $B(\delta)$ but not through the vertical box $V$ by the definition of $H_{0}$. Hence, the first exiting point of $\Pi^{n}\left(z^{\prime}\right)$ does not belong to any one of the $U_{i}$ 's for $1 \leq i \leq M$. This contradicts $\Pi^{n}\left(z^{\prime}\right) \in \Lambda(F, U)$. The other case with $\Pi^{m}\left(z^{\prime}\right)$ is identical.

Proof of Theorem 1.4. We must, of course, define a topological conjugacy, say $\rho: \Sigma\left(K_{0}\right) \rightarrow \Lambda(F, U)$. We actually start by defining a map $\rho: \bigcup_{n \in \mathbf{Z}} \sigma^{n}(\widehat{\mathscr{S}}) \rightarrow$ $\bigcup_{n \in \mathbf{Z}} F^{n}(\bar{\Omega})$ and showing that it is a conjugacy, where $\widehat{\mathscr{S}}$ is the standard blowup as in (1.4b). Then, the one-point compactification will naturally take care of the rest of the proof.

As the first preparation, we point out that the map $\psi$ defined in Lemma 2.3 with $\mathscr{A}=\bar{\Omega}$ is actually homeomorphic. This is because $\bar{\Omega}$ is compact, $\widehat{\mathscr{S}}$ is Hausdorff, and the inverse $\psi^{-1}$ is continuous.

As another preparation, we examine the operation of blowups in finer detail. It is easy to see by definition that, up to only renaming the subscripts, every element $\hat{s}$ from $\bigcup \sigma^{n}(\widehat{\mathscr{S}})$ can be written as $\hat{s}=\hat{s}(l):=\left(\cdots \hat{s}_{-1} \hat{s}_{0, l} \hat{s}_{1} \cdots\right)$ for some $0 \leq l<s_{0}-1$ with the properties that $\hat{s}_{0, l}=s_{0}^{-l} \cdots s_{0}^{0} \cdots s_{0}^{s_{0}-1-l}$ if $s_{0} \neq \infty^{\mathrm{s}}, \infty^{\mathrm{u}}$ or $\hat{s}_{0, l}=\infty^{\mathrm{s}}, \infty^{u}$ and $l=0$ otherwise; and $\hat{s}_{i}=s_{i}^{0} \cdots s_{i}^{s_{i}-1}$ if $i \neq 0$ with $s_{i} \neq \infty^{\mathrm{s}}, \infty^{\mathrm{u}}$, or $\hat{s}_{i}=\infty^{\mathrm{s}}, \infty^{\mathrm{u}}$ otherwise. Recall that $\hat{s}(l)$ is called a standard blowup only if $l=0$, or, if $s_{0}$ is not finite, it must be the first $\infty^{\mathrm{s}}$, namely $s_{-1}$ is finite. In other words, $\hat{s}=\left(\cdots \hat{s}_{-1} s_{0}^{0} s_{0}^{1} \cdots s_{0}^{s_{0}-1} \cdots\right)$ and $\hat{s}=\left(\cdots s_{-1}^{0} \cdots s_{-1}^{s_{-1}-1} \infty_{0}^{s} \infty_{1}^{s} \cdots\right)$ are the only two types of standard blowups. 
As the last preparation, we introduce the standard backward iteration $\tau_{b}$ for all $\hat{s} \in \bigcup \sigma^{n}(\widehat{\mathscr{S}})$ as follows:

$$
\tau_{b}(\hat{s})= \begin{cases}-l, & \text { if } \hat{s}=\hat{s}(l) \text { and } s_{0} \neq \infty^{\mathrm{s}}, \infty^{\mathrm{u}}, \\ n+1, & \text { if } s_{0}=\infty^{\mathrm{u}} \text { and } \hat{s}=\left(\cdots \infty_{0}^{\mathrm{u}} \cdots \infty_{n}^{\mathrm{u}} \hat{s}_{n+1} \cdots\right), \\ m, & \text { if } s_{0}=\infty^{\mathrm{s}} \text { and } \hat{s}=\left(\cdots \hat{s}_{m-1} \infty_{m}^{\mathrm{s}} \cdots \infty_{0}^{\mathrm{s}} \cdots\right)\end{cases}
$$

Roughly speaking, $\sigma^{\tau_{b}(\cdot)}(\cdot)$ maps nonstandard blowups back into standard ones by the minimum number of 'backward' shifts. Moreover, the same definition applies to all $z \in \bigcup F^{n}(\bar{\Omega})$. To be precise, we have

$$
\tau_{b}(z)= \begin{cases}-l, & \text { the least backward iterations so that } F^{-l}(z) \in \Omega, \\ n+1, & \text { the least forward iterations so that } F^{n+1}(z) \in W_{h}^{\mathrm{u}}, \\ m, & \text { the least backward iterations so that } F^{m}(z) \in W_{H}^{\mathrm{s}}\end{cases}
$$

For this reason, we will not distinguish these two functions. Similarly, one can define the standard forward iteration $\tau_{f}$ as

$$
\tau_{f}(\hat{s})= \begin{cases}s_{0}-l, & \text { if } \hat{s}=\hat{s}(l) \text { and } s_{0} \neq \infty^{\mathrm{s}}, \infty^{\mathrm{u}}, \\ n+1, & \text { if } s_{0}=\infty^{\mathrm{u}} \text { and } \hat{s}=\left(\cdots \infty_{0}^{\mathrm{u}} \cdots \infty_{n}^{\mathrm{u}} \hat{s}_{n+1} \cdots\right), \\ m, & \text { if } s_{0}=\infty^{\mathrm{s}} \text { and } \hat{s}=\left(\cdots \hat{s}_{m-1} \infty_{m}^{\mathrm{s}} \cdots \infty_{0}^{\mathrm{s}} \cdots\right) .\end{cases}
$$

It is trivial to see that $\tau_{f}(\hat{s})=\tau_{b}(\hat{s})+s_{0}$ if $s_{0} \neq \infty^{\mathbf{s}}, \infty^{\mathbf{u}}$ and $\tau_{f}(\hat{s})=\tau_{b}(\hat{s})$ otherwise. The extension of $\tau_{f}$ to $\bigcup F^{n}(\bar{\Omega})$ is identical to $\tau_{b}$ above. The important property we will use in what follows is

$$
\tau_{b}(\sigma(\hat{s}))= \begin{cases}\tau_{b}(\hat{s})-1, & \text { if } \hat{s}=\hat{s}(l), l<s_{0}-1, \text { or } s_{0}=\infty^{\mathbf{s}}, \infty^{\mathbf{u}}, \\ \tau_{f}(\hat{s})-1, & \text { if } l=s_{0}-1 .\end{cases}
$$

This can be directly checked by definition.

We now define

$$
\rho(\hat{s}):=F^{-\tau_{b}(\hat{s})}\left(\psi\left(\sigma^{\tau_{b}(\hat{s})}(\hat{s})\right)\right) .
$$

We need to show it is (1) one-to-one, (2) onto, (3) commutative with $F$ and $\sigma$, and (4) homeomorphic. We start with the one-to-one property. Let $\hat{s}$, $\hat{s}^{\prime} \in \bigcup \sigma^{n}(\widehat{\mathscr{S}})$ and suppose $z=\rho(\hat{s})=\rho\left(\hat{s}^{\prime}\right)=z^{\prime}$. Then, by definitions $(3.5 \mathrm{~b})$ and (3.7), $\tau_{b}(z)$ must be $\tau_{b}(\hat{s})$. This implies $\tau_{b}(\hat{s})=\tau_{b}(z)=\tau_{b}\left(z^{\prime}\right)=\tau_{b}\left(\hat{s}^{\prime}\right)$. Therefore, $\psi\left(\sigma^{\tau_{b}(\hat{s})}(\hat{s})\right)=\psi\left(\sigma^{\tau_{b}(\hat{s})}\left(\hat{s}^{\prime}\right)\right) \in \mathscr{A}$ because $F$ is homeomorphic. Since $\psi$ is homeomorphic, $. \sigma^{\tau_{b}(\hat{s})}(\hat{s})=\sigma^{\tau_{b}\left(\hat{s}^{\prime}\right)}\left(\hat{s}^{\prime}\right)$. That is, $\hat{s}$ and $\hat{s}^{\prime}$ give rise to the same standard blowup. Since two elements of $\bigcup \sigma^{n}(\widehat{\mathscr{S}})$ which have the same standard blowup are different only by the 'minimum' time of standard backward iterations, we can conclude $\hat{s}=\hat{s}^{\prime}$ because $\tau_{b}(\hat{s})=\tau_{b}\left(\hat{s}^{\prime}\right)$ as shown above.

Second, we show $\rho$ is onto. This simply follows the reversal definition of (3.7). To be precise, for a given $z \in \bigcup F^{n}(\bar{\Omega})$, let $\tau_{b}(z)$ be its standard backward iteration. Thus $F^{\tau_{b}(z)}(z) \in \bar{\Omega}$. Blow up the corresponding sequence and obtain $\psi^{-1} \circ F^{\tau_{b}(z)}(z)$. Then apply $-\tau_{b}(z)$ shifts $\sigma^{-\tau_{b}(z)}$. The resulting nonstandard blowup $\hat{s}$ is what we look for because it is trivial to check by the definitions $\tau_{b}(\hat{s})=\tau_{b}(z)$ and $\rho(\hat{s})=z$. 
To show the commutativity and the homeomorphism property, we need the following alternative definition of $\rho$ in terms of the 'minimum' standard forward iteration. We show, indeed,

$$
\rho(\hat{s})=F^{-\tau_{f}(\hat{s})}\left(\psi\left(\sigma^{\tau_{f}(\hat{s})}(\hat{s})\right)\right) .
$$

When $s_{0}=\infty^{\mathrm{s}}, \infty^{\mathrm{u}},(3.8)$ immediately follows from (3.5a) and (3.5c) because of $\tau_{b}(\hat{s})=\tau_{f}(\hat{s})$. Suppose $s_{0} \neq \infty^{\mathrm{s}}, \infty^{\mathrm{u}}$. Then, (3.8) follows from the following facts:

(i) $\tau_{f}(\hat{s})=\tau_{b}(\hat{s})+s_{0}$ by $(3.5 \mathrm{a})$ and $(3.5 \mathrm{c})$;

(ii) $\psi\left(\sigma^{s_{0}}(\hat{s})\right)=F^{s_{0}}(\psi(\hat{s}))$, that is, $\psi\left(\sigma^{s_{0}}(\hat{s})\right)$ is equal to the Poincare return of $\psi(\hat{s})$ which, by definition, takes exactly $s_{0}$ iterates of $F$.

We now have

$$
\begin{aligned}
F^{-\tau_{f}}\left(\psi\left(\sigma^{\tau_{f}}(\hat{s})\right)\right) & =F^{-\tau_{f}}\left(\psi \circ \sigma^{s_{0}}\left(\sigma^{\tau_{b}}(\hat{s})\right)\right)=F^{-\tau_{f}}\left(F^{s_{0}} \circ \psi\left(\sigma^{\tau_{b}}(\hat{s})\right)\right) \\
& =F^{-\tau_{b}}\left(\psi\left(\sigma^{\tau_{b}}(\hat{s})\right)\right)=\rho(\hat{s}) .
\end{aligned}
$$

We now show the commutativity, using (3.6) and (3.8). When $l \neq s_{0}-1$, it is straightforward to have

$$
\begin{aligned}
\rho \circ \sigma(\hat{s}) & =F^{-\tau_{b}(\sigma(\hat{s}))}\left(\psi\left(\sigma^{\tau_{b}(\sigma(\hat{s}))} \circ \sigma(\hat{s})\right)\right) \\
& =F\left(F^{-\tau_{b}(\hat{s})}\left(\psi\left(\sigma^{\tau_{b}(\hat{s})-1} \circ \sigma(\hat{s})\right)\right)\right)=F \circ \rho(\hat{s}) .
\end{aligned}
$$

On the other hand, when $l=s_{0}-1$, we replace $\tau_{b}(\sigma(\hat{s}))$ by $\tau_{f}(\hat{s})-1$. Then the equivalent definition (3.8) together with the same computation above applies.

Next, we show $\rho$ is homeomorphic. To this end, let $m$ and $n$ be the least and largest number such that $s_{m}=\infty^{\mathrm{s}}$ and $s_{n}=\infty^{\mathrm{u}}$ respectively for a given segment $\hat{s}_{-l} \cdots \hat{s}_{0} \cdots \hat{s}_{k}$. Let $b>\max \{|n|,|m|\}$. Let $B_{\hat{s}_{-l} \cdots \hat{s}_{k}}$ be such a typical topological basis element for $U \sigma^{n}(\widehat{\mathscr{S}})$ that consists of those $\hat{s}^{\prime}$ such that $\hat{s}_{i}^{\prime j}=\hat{s}_{i}^{j}$ for all $i$ and $j$ or only for those $i$ with $s_{i} \neq \infty^{\mathrm{s}}$ or $\infty^{\mathrm{u}}$, but $s_{i}^{\prime j} \geq b$, where, recall, $\hat{s}_{i}=s_{i}^{0} \cdots s_{i}^{s_{i}-1}$, etc. We claim first that

$$
\begin{array}{ll}
\tau_{b}\left(B_{\hat{s}_{-} \cdots \hat{s}_{k}}\right)=\text { constant, } & \text { if } s_{0} \neq \infty^{\mathrm{u}}, \\
\tau_{f}\left(B_{\hat{s}_{-}, \cdots \hat{s}_{k}}\right)=\text { constant, } & \text { if } s_{0} \neq \infty^{\mathrm{s}} .
\end{array}
$$

If $s_{0} \neq \infty^{\mathrm{s}}, \infty^{\mathrm{u}}$, then it is trivial by definitions (3.5a) and (3.5c) and the constant is $\tau(\hat{s})$. Suppose $s_{0}=\infty^{\mathrm{s}}$. Then, it is easy to see from the definition of $b$ that for every $\hat{s}^{\prime} \in B_{\hat{s}_{-} \cdots \hat{s}_{k}}$, either $s_{0}^{\prime}=\infty^{\mathrm{s}}$ so that $\tau_{b}\left(\hat{s}^{\prime}\right)=m$ or $s_{0}^{\prime} \neq \infty^{\mathrm{s}}$, but then the blowup $\hat{s}_{i}^{\prime}$ must spread across the segment from the $m$ th place to the initial place since $s_{i}^{\prime} \geq b \geq|m|$ for $m \leq i$. That is, $\hat{s}^{\prime}=\left(\cdots \hat{s}_{m-1} s_{0}^{m} \cdots s_{0}^{0} \cdots\right)$ and $\tau_{b}\left(\hat{s}^{\prime}\right)$ must be $m$. The other case where the standard forward iteration must be constant for $s_{0} \neq \infty^{s}$ is identical. This proves the claim. Now, depending on whether $s_{0}=\infty^{\mathrm{s}}$ or $\infty^{\mathrm{u}}$, we use either $\tau_{b}$ or $\tau_{f}$ in the definition of $\rho$ so that $\tau_{b}(B)$ or $\tau_{f}(B)$ is constant. Then, the openness of $\rho$ follows that of $\sigma^{\tau}$ for a fixed $\tau$, together with the openness of $\psi$ by Lemma 2.3 and $F^{-\tau}$ for a fixed $\tau$. This shows that the inverse $\rho^{-1}$ is continuous. The continuity of $\rho$ is similarly obvious by its definition together with the continuity of $F$, $\psi, \sigma$, and the identity (3.9).

Finally, taking the one-point compactification for $U \sigma^{n}(\widehat{\mathscr{S}})$ and $\bigcup F^{u}(\bar{\Omega})$ simultaneously results in the natural homeomorphic extension for $\rho$.

The proof of Theorem 1.6 is trivial. We omit it. 
As a final remark for this section, let us point out that the proofs of Theorems 1.3 and 1.4 above do not use the hyperbolicity of the fixed point $q$. Thus, the conclusion as well as the proof of Theorem 1.4 also holds true so long as $(2.5 \mathrm{~b})$ is satisfied. This is precisely the case for the nonhyperbolic fixed point which we will pursue in the next section.

\section{THE PROOF OF THEOREM 1.7}

The key step in proving this theorem is to verify conditions $(2.5 b)$ and (2.7). But, by means of the exponential expansion (Proposition 4.1) from our previous project, this in turn reduces to the asymptotic analysis on the local center manifold (Lemma 4.2).

To begin with, let the local coordinates $x:=\left(x_{c}, x_{s}\right)$ and $y$ be chosen so that $W^{\mathrm{cs}}(\alpha)=\{y=0\}, W^{\mathrm{cu}}(\alpha)=\left\{x_{s}=0\right\}$, and $W^{\mathrm{c}}(\alpha)=\left\{x_{s}=0, y=0\right\}$ locally. Here, the parameter is explicitly included, as it is for $F=F(z, \alpha)$. Let us also use $F=\left(F_{c}, F_{s}, F_{u}\right)$ for the componentwise form. Then, the coordinate is said to be admissible if, in addition, $F_{c}\left(x_{c}, 0, y, \alpha\right)=F_{c}\left(x_{c}, x_{s}, 0, \alpha\right):=$ $f_{c}\left(x_{c}, \alpha\right)$. Note that an admissible coordinate directly gives rise to 'straight' invariant foliations on the center stable and center unstable manifolds as $W^{\mathrm{cs}}=$ $\bigcup_{\left|x_{c}\right| \ll 1}\left\{x=x_{c}, y=0\right\}$ and $W^{\mathrm{cu}}=\bigcup_{\left|x_{c}\right| \ll 1}\left\{x=x_{c}, x_{s}=0\right\}$ respectively. The admissible coordinate will be mentioned later in $\S 5$. Referring our readers to Deng (1988b) for its proof, we now state the following result.

Proposition 4.1. There exists a $C^{r-2}$ admissible coordinate such that $f_{c}$ is $C^{r}$ and the local orbits admit a $C^{r-3}$ exponential expansion in the following sense. Let $n_{c}\left(x_{c}, 0\right)$ be the local life span of a center orbit, i.e., $n_{c}=$ $\max \left\{k:\left|f_{c}^{k}\left(x_{c, 0}\right)\right| \leq \delta, k \geq 0\right\}$. Then, for every small $\delta>0$ there is a constant $C_{1}$ such that for all $0 \leq l \leq n_{c},\left|x_{c, 0}\right| \leq \delta,\left|x_{s, 0}\right| \leq \delta,\left|y_{1}\right| \leq \delta$, and $|\alpha| \leq \delta$ there is a unique orbit

$$
\gamma=\left\{\left(x^{n}, y^{n}\right)=F^{n}\left(x^{0}, y^{0}, \alpha\right): 0 \leq n \leq l\right\} \subset B\left(C_{1} \delta\right)
$$

satisfying $\left(x_{c}^{0}, x_{s}^{0}\right)=\left(x_{c, 0}, x_{s, 0}\right)$ and $y^{l}=y_{1}$.

Moreover, if we designate this correspondence as

$$
\left(x_{c}^{n}, x_{s}^{n}, y^{n}\right)=\left(x_{c}^{n}, x_{s}^{n}, y^{n}\right)\left(l, x_{c, 0}, x_{s, 0}, y_{1}, \alpha\right),
$$

then it is $C^{r-3}$ in $x_{0}, y_{1}$, and $\alpha$. Furthermore, there exist constants $0<\lambda<$ $1<\mu$ and $C_{2}$ so that

$$
\begin{gathered}
\left\|x_{s}^{n}(l, \cdot, \cdot, \cdot, \cdot)\right\|_{r-3} \leq C_{2} \lambda^{n}, \quad\left\|y^{n}(l, \cdot, \cdot, \cdot, \cdot)\right\|_{r-3} \leq C_{2} \mu^{n-l}, \\
\left\|x_{c}^{n}(l, \cdot, \cdot, \cdot, \cdot)-x_{c}^{n}(l, \cdot, 0,0, \cdot)\right\|_{r-3} \leq C_{2} \lambda^{n} \mu^{n-l},
\end{gathered}
$$

for all $0 \leq n \leq l$, where $\|\cdot\|_{r-3}$ denotes the $C^{r-3}$ norm.

With this result, the validity of condition $(2.5 \mathrm{~b})$ is straightforward. Indeed, restricting the variables $\left(x_{0}, 0\right),\left(0, y_{1}\right)$ to the boxes $B\left(\delta_{i}, p_{i}\right)$ for $i=1,2$ respectively and letting $k=l+M+1$, we have, similarly to (3.1a),

$$
\begin{aligned}
\hat{x}_{c}\left(k, x_{c, \text { in }}, x_{s, \text { in }}, y_{\text {out }}, \alpha\right) & :=x_{c}^{l}\left(l, x_{c, 0}, x_{s, 0}, y_{1}, \alpha\right), \\
\hat{x}_{s}\left(k, x_{c, \text { in }}, x_{s, \text { in }}, y_{\text {out }}, \alpha\right) & :=x_{s}^{l}\left(l, x_{c, 0}, x_{s, 0}, y_{1}, \alpha\right), \\
\hat{y}\left(k, x_{c, \text { in }}, x_{s, \text { in }}, y_{\text {out }}, \alpha\right) & :=y^{0}\left(l, x_{c, 0}, x_{s, 0}, y_{1}, \alpha\right) .
\end{aligned}
$$


To verify condition (2.7), recall the Lipschitz constant $L$ for $\hat{x}$ and $\hat{y}$ from Lemma 2.1. It is easy to see from the exponential expansion estimates (4.1) that in order for $L$ to be small, it suffices to show that the center Lipschitz constant $\operatorname{Lip} \hat{x}_{c}\left(k, x_{c, \text { in }}, 0,0, \alpha\right)$ is small. Since the Lipschitz constant $g$ for the global map $\Pi_{g}$ from Lemma 2.1 is independent of $\delta_{1}$, to show $g L$ is small it suffices to show

$$
\text { Lip } \hat{x}_{c}\left(k, x_{c, \text { in }}, 0,0, \alpha\right) \leq C_{3} \delta_{1}
$$

from some constant $C_{3}$ depending on $\delta$ and $\delta_{0}$ only. To specify the iteration time $k$ for which (4.3a) holds, we have

Lemma 4.2. Let $\delta, \delta_{0}, \delta_{1}$ be small constants as in Lemma 2.1 and Proposition 4.1 above. Let

$$
\begin{aligned}
& K_{0}=\max \left\{\min \left\{k: \hat{x}_{c}\left(k, x_{c, \text { in }}, 0,0, \alpha\right) \leq \delta_{1}\right\}:\left|x_{c, \text { in }}-x_{0}\right| \leq \delta_{0},|\alpha| \leq \delta\right\}, \\
& K_{1}=K_{1}(\alpha)=\left\{\begin{array}{rr}
\min \left\{\max \left\{k: \hat{x}_{c}\left(k, x_{c}, \text { in }, 0,0, \alpha\right) \geq 0\right\}: \mid x_{c} \text { in }-x_{0} \mid \leq \delta_{0}\right\}, \\
\alpha<0, \\
\infty, \quad \alpha \geq 0,
\end{array}\right. \\
& K_{2}=K_{2}(\alpha)=\left\{\begin{array}{rr}
\max \left\{\max \left\{k: x_{c}\left(k, x_{c}, \text { in }, 0,0, \alpha\right) \geq-\delta_{1}\right\}:\left|x_{c, \text { in }}-x_{0}\right| \leq \delta_{0}\right\}, \\
\infty, & \alpha \geq 0 .
\end{array}\right.
\end{aligned}
$$

Then, there are constants $C_{3}=C_{3}\left(\delta, \delta_{0}\right), C_{0}=C_{0}\left(\delta, \delta_{0}, \delta_{1}\right)$, and $\varepsilon_{0}=$ $\varepsilon_{0}\left(\delta, \delta_{0}, \delta_{1}\right)$ such that (4.3a) holds for all $K_{0} \leq k \leq K_{1}(\alpha),\left|x_{c, \text { in }}-x_{0}\right| \leq \delta_{0}$, and $|\alpha| \leq \varepsilon_{0}$. Moreover,

$$
K_{1}(\alpha) \leq K_{2}(\alpha) \leq \frac{C_{0}}{\sqrt{|\alpha|}} \quad \text { and } \quad K_{1}(\alpha) \rightarrow+\infty \quad \text { as } \alpha \rightarrow 0^{-} .
$$

Proof. Since the proof has nothing to do with the other components $x_{s}, y$, we will simplify our notation by letting $x=x_{c}, x_{c}$, in $=x^{0}$ with $\left|x^{0}-x_{0}\right| \leq \delta_{0}$ and $\hat{x}_{c}\left(k, x_{c}\right.$,in $\left., 0,0, \alpha\right)=f_{c}^{l}\left(x^{0}, \alpha\right)$. Recall that $k=l+M+1$ and $M$ is the number of distinct points of $\gamma(p)$ outside $B(\delta)$. Also, because of the assumptions $(1.5 \mathrm{a}, \mathrm{b}, \mathrm{c})$ on the parameter $\alpha$ for the saddle-node bifurcation, we may assume, without loss of generality,

$$
f_{\mathcal{c}}(x, \alpha):=\alpha+x-f(x, \alpha)
$$

with the property that $f_{x}(0, \alpha)=0, f_{x x}(0, \alpha)>0$. More specifically, we assume, up to rescaling, that

$$
f_{x}(0, \alpha)=0, \quad f_{x x}(x, \alpha) \geq 4, \quad|x| \leq \delta .
$$

This implies

$$
f(x, \alpha) \geq 2 x^{2} \text { and } f_{c} \leq \alpha+x-x^{2} .
$$

Now, our proof will be split into two cases for $\alpha \geq 0$ and $\alpha<0$.

The $\alpha \geq 0$ case. First, we find the fixed point $x^{+}$which is to the right of the origin. Solving $f_{c}(x, \alpha)=x$ by the implicit function theorem, we obtain a $C^{r}$ function $\alpha=\alpha(x)$ for $|x| \leq \delta$ and $\delta \ll 1$. Indeed, the two branches $(x>0, x<0)$ of fixed points are in one-to-one correspondence with $\alpha$ in the following way

$$
\pm \sqrt{\alpha}=\sqrt{\frac{1}{2} f_{x x}(0, \alpha)+O(|x|) x} .
$$


Hence, if we let $\varepsilon=\sqrt{\alpha}$, then by the implicit function theorem again, $x^{+}(\varepsilon)$ can be solved as a $C^{r-2}$ function of $\varepsilon$ from the equation above and $x^{+}(\varepsilon)=O(\varepsilon)$.

By making the change of variable $x \rightarrow \bar{x}+x^{+}(\varepsilon)$, the map under the new variable $\bar{x}$ takes the form

$$
\bar{f}_{c}:=f_{c}\left(\bar{x}+x^{+}(\varepsilon), \varepsilon^{2}\right)-x^{+}(\varepsilon)=\varepsilon^{2}+\bar{x}-f\left(\bar{x}+x^{+}(\varepsilon), \varepsilon^{2}\right) .
$$

Using Taylor expansion and $\varepsilon^{2}=f\left(x^{+}(\varepsilon), \varepsilon^{2}\right)$, we have $\bar{f}_{c}(\bar{x}, \alpha)=\lambda(\varepsilon) \bar{x}-$ $f_{1}(\bar{x}, \varepsilon)$, where $\lambda(\varepsilon)=1-f_{x}\left(x^{+}(\varepsilon), \varepsilon^{2}\right) \leq 1$ since $f_{x}\left(x^{+}(\varepsilon), \varepsilon^{2}\right) \geq 0$ for $f_{x x} \geq 0$ and $x^{+}(\varepsilon) \geq 0$. Also

$$
f_{1}(\bar{x}, \varepsilon):=f\left(\bar{x}+x^{+}(\varepsilon), \varepsilon^{2}\right)-f\left(x^{+}(\varepsilon), \varepsilon^{2}\right)-f_{x}\left(x^{+}(\varepsilon), \varepsilon^{2}\right) \bar{x} .
$$

We suppress the bars from the new variable in the following. By elementary calculus together with $f_{x}(0, \alpha) \geq 0$ and $(4.4 \mathrm{~b})$, it is easy to show that

$$
f_{1}(x, \varepsilon) \geq 2 x^{2} \text { and } \bar{f}_{c}(x, \varepsilon) \leq x-x^{2} .
$$

Let $x^{l}=\bar{f}_{c}^{l}\left(x^{0}, \alpha\right)$ and $y^{l}=\tilde{f}^{l}\left(y^{0}\right)$ with $\tilde{f}=y-y^{2}$. Then, motivated by the comparison principle for continuous flows together with the monotonicity of $\bar{f}_{c}(\cdot, \alpha)$ and $\tilde{f}$, one can easily show

$$
x^{l} \leq y^{l} \quad \text { if } x^{0} \leq y^{0} .
$$

See Figure 4.1. Since $x^{l}, y^{l}$ are monotone decreasing because $x^{l}-x^{l-1}=$ $-f_{x}\left(x^{+}(\varepsilon), \varepsilon\right)-f_{1}\left(x^{l-1}, \varepsilon\right)<0$ and $y^{l}-y^{l-1}=-\left(y^{l-1}\right)^{2}<0$, and positive because $x^{0}>0$ and $y^{0}>0$, they must converge to the fixed point $x=0$.

To estimate the rate of convergence, let, specifically, $y^{0}=x_{0}+\delta_{0}$ be the right end point of $\left|x^{0}-x_{0}\right| \leq \delta_{0}$, and let $y^{l}$ be the corresponding upper orbit. Then we have $x^{l} \leq y^{l}$ for all $\left|x^{0}-x_{0}\right| \leq \delta_{0}$. Thus, for the old variable, $\left|x^{l}\right|=\left|\bar{x}^{l}+x^{+}(\varepsilon)\right| \leq \delta_{1}$ is satisfied uniformly for all $l \geq K_{0}\left(\delta, \delta_{0}, \delta_{1}\right)-M-1$ and $|\alpha| \leq \varepsilon_{0}=\varepsilon_{0}\left(\delta, \delta_{0}, \delta_{1}\right)$ with some appropriately chosen $K_{0}$ and $\varepsilon_{0}$ so that $y^{l} \leq \delta_{1} / 2$.

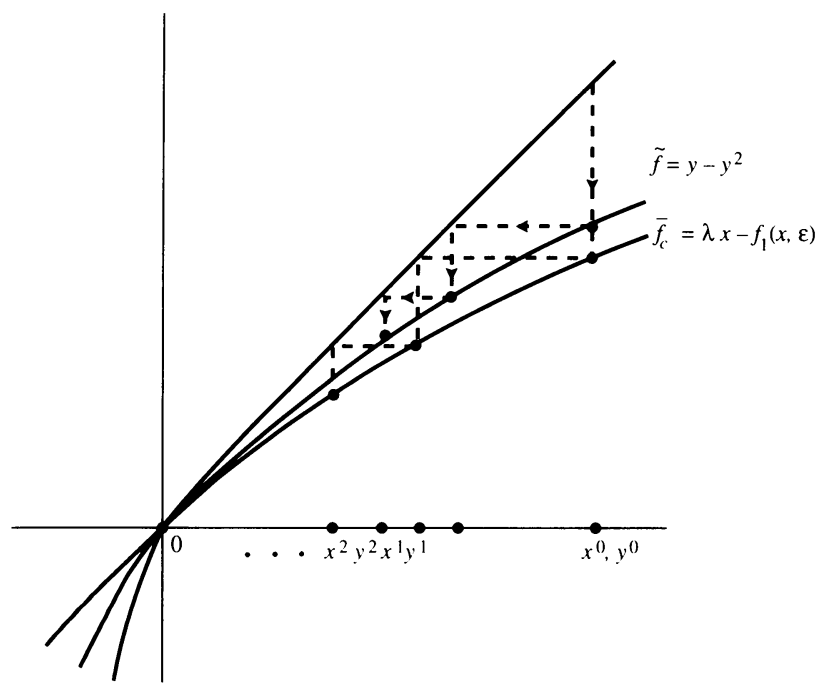

FIGURE 4.1 
Next, to show $D_{x} x^{l}\left(x^{0}, \alpha\right) \rightarrow 0$ as $k \rightarrow \infty$ uniformly for $\left|x^{0}-x_{0}\right| \leq \delta_{0}$ in the old variable, we show the same thing for the new variable instead since $D_{\bar{x}} \bar{x}^{l}\left(\bar{x}^{0}, \alpha\right)=D_{x} x^{l}\left(x^{0}, \alpha\right)$. Again, we suppress the bars from the $x$-variable for simplicity of notation. By the chain rule, we have

$$
D x^{l}=\prod_{n=0}^{l-1}\left[\lambda(\varepsilon)-f_{1 x}\left(x^{n}, \varepsilon\right)\right] \leq \prod_{n=0}^{l-1}\left[\lambda(\varepsilon)-\frac{f_{1}\left(x^{n}, \varepsilon\right)}{x^{n}}\right]:=I_{l},
$$

where we have used $f_{1 x}(x, \varepsilon) \geq f_{1}(x, \varepsilon) / x$ which can be easily proved by elementary calculus together with the definitions (4.5a) and (4.4b). Observe

$$
\begin{aligned}
x^{l} & =\lambda(\varepsilon) x^{l-1}-f_{1}\left(x^{l-1}, \varepsilon\right) \\
& =\left(\lambda(\varepsilon)-\frac{f_{1}\left(x^{l-1}, \varepsilon\right)}{x^{l-1}}\right) x^{l-1}=I_{l} \cdot x^{0} .
\end{aligned}
$$

This implies $I_{l}=x^{l} / x^{0} \leq y^{l} /\left(x_{0}-\delta_{0}\right) \leq C_{3} \delta_{1}$ and therefore implies (4.3a) for an appropriately chosen $C_{3}$.

The $\alpha<0$ case. We show the estimate (4.3b) first, and then the rest of the proof is similar in the same spirit as in the $\alpha \geq 0$ case above. The idea of proof is motivated by the comparison between the orbit $\gamma\left(x^{0}\right)$ and the solution $y\left(t, x^{0}, \alpha\right)$ of the equation $\dot{y}=\alpha-y^{2}$. It is inspired by the fact that $x^{l+1}-x^{l}=$ $\alpha-f\left(x^{l}, \alpha\right) \leq \alpha-\left(x^{l}\right)^{2}$. To be precise, we claim

$$
\begin{aligned}
y(-1, x, \alpha) \leq f_{c}^{-1}(x, \alpha), & \text { for either } x \geq f_{c}(0, \alpha)=\alpha, \\
& \text { or } x \leq f_{c}^{3}(0, \alpha) .
\end{aligned}
$$

That is, a unit time backward integration of $\dot{y}=\alpha-y^{2}$ is always bounded from above by the backward iterate of $f_{c}$ in the shown region. To proceed, let $f_{c}(x, \alpha)$ substitute for $x$ and $\alpha=-\varepsilon^{2}$. Then $(4.7 \mathrm{a})$ reads

$$
y\left(-1, f_{c}(x, \alpha), \alpha\right) \leq x, \quad \text { for } x \geq 0 \text {, or } x \leq f_{c}^{2}(0, \alpha) \leq-2 \varepsilon^{2} .
$$

Here, the last inequality $f_{c}^{2}(0, \alpha)=f_{c}\left(-\varepsilon^{2}, \alpha\right) \leq-2 \varepsilon^{2}$ is trivial, where $f_{c}^{2}$ means the second iterate of $f_{c}$. One can directly check by differentiation that

$$
y(t, x, \varepsilon)=(x-\varepsilon \tan \varepsilon t)\left[1+x \frac{\tan \varepsilon t}{\varepsilon}\right]^{-1} .
$$

Thus, $(4.7 \mathrm{~b})$ is equivalent to $e(x, \varepsilon) \leq 0$, where

$$
e(x, \varepsilon):=f_{c}(x, \alpha)+\varepsilon \tan \varepsilon-x\left(1-f_{c}(x, \varepsilon) \frac{\tan \varepsilon}{\varepsilon}\right) .
$$

Recall $f_{c}(x, \alpha) \leq-\varepsilon^{2}+x-2 x^{2}$ since $f(x, \alpha) \geq 2 x^{2}$ and $\tan \varepsilon=\varepsilon-\frac{1}{3} \varepsilon^{3}+$ $O\left(\varepsilon^{5}\right)$. We have

$$
\begin{aligned}
e(x, \varepsilon) \leq & -\varepsilon^{2}+x-2 x^{2}+\varepsilon\left(\varepsilon-\frac{1}{3} \varepsilon^{3}+O\left(\varepsilon^{5}\right)\right) \\
& -x\left\{1-\left[-\varepsilon^{2}+x-f(x, \varepsilon)\right] \frac{\tan \varepsilon}{\varepsilon}\right\} \\
= & -x^{2}\left(2-\frac{\tan \varepsilon}{\varepsilon}\right)-\frac{1}{3} \varepsilon^{4}+O\left(\varepsilon^{6}\right)-x\left[\varepsilon^{2}+f(x, \varepsilon)\right] \frac{\tan \varepsilon}{\varepsilon} \\
= & -x^{2}\left[2-\frac{\tan \varepsilon}{\varepsilon}+\left(\frac{\varepsilon^{2}}{x}+\frac{f(x, \varepsilon)}{x}\right) \frac{\tan \varepsilon}{\varepsilon}\right]-\frac{1}{3} \varepsilon^{4}+O\left(\varepsilon^{6}\right) .
\end{aligned}
$$


It is trivial to see that $e(x, \varepsilon)<0$ when $x>0$. It is also true when $x \leq-2 \varepsilon^{2}$ since the leading term of the bracket is greater than $\frac{1}{2}+f(x, \varepsilon) / x=O(|x|)$ and $|x| \leq \delta$.

We now show (4.3b). The limit is trivial. To show the estimate, we begin with $K_{1}$. Let $n=K_{1}-(M+1)$. Then by definition, $x^{l} \geq x^{n} \geq 0$ for $l \leq n$. Hence, successively applying the claim $(4.7 \mathrm{~b})$ together with the monotonicity of $y(t, x, \alpha)$ in $t$ and the monotonicity of $x^{l}$ in $l$, we have $0<x^{n} \leq$ $y\left(-l, x^{n}, \alpha\right) \leq x^{n-l}$ (cf. Figure 4.2). Since the numerator of $y$ in (4.8) is positive all the time for $t<0$, the denominator will not change its sign. Thus $1-x^{n} \tan \varepsilon n / \varepsilon \geq 0$, implying

$$
K_{1} \leq \frac{1}{\varepsilon} \tan ^{-1} \frac{\varepsilon}{x^{n}}+M+1 \leq \frac{1}{\varepsilon} \tan ^{-1} \frac{1}{\varepsilon}+M+1 .
$$

The estimate for $K_{2}$ is similar. Indeed, let $x_{0}$ be such that $f_{c}^{k}\left(x_{0}, \alpha\right) \geq-\delta_{1}$ for $k \leq K_{2}-(M+1):=n$ and let $k_{1}:=\max \left\{k: f_{c}^{k}\left(x_{0}, \alpha\right) \geq 0\right\}$. Thus $x^{l}=f_{c}^{l}\left(x_{0}, \alpha\right) \leq f_{c}^{2}(0, \alpha)$ for all $l \geq k_{1}+3$ and (4.7b) applies. Hence, tracing backward $n-k_{1}-3$ times we still have $x^{n} \leq y\left(-n+k_{1}+3, x^{n}, \alpha\right) \leq 0$ (cf. Figure 4.2 also). This time, however, the numerator of $y$ remains constant in signs instead. Therefore, $x^{n}+\varepsilon \tan \varepsilon\left(n-k_{1}-3\right) \leq 0$. This implies

$$
n \leq \frac{1}{\varepsilon} \tan \frac{\left|x^{n}\right|}{\varepsilon}+k_{1}+3=O\left(\frac{1}{\varepsilon}\right),
$$

since $k_{1}$ also has the order of $\frac{1}{\varepsilon}$ by what has just been proved above for the case $K_{1}$. This completes the proof for $(4.3 \mathrm{~b})$.
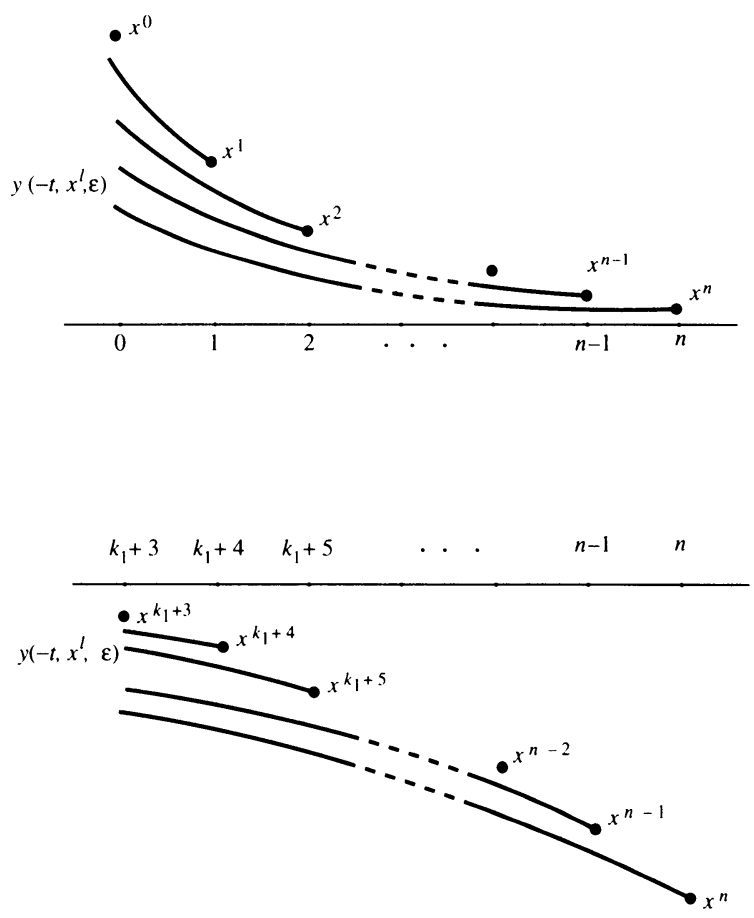

FIGURE 4.2 
Next, the remaining proof is similar to the $\alpha \geq 0$ case above. The constant $K_{0}$ is obtained from the relation $x^{l} \leq y^{l} \leq \delta_{1}$ for $l \geq K_{0}-M-1$ with $y^{l}$ the upper orbit with $y^{l+1}=y^{l}-\left(y^{l}\right)^{2}$ and $y^{0}=\delta_{0}+x_{0}$. Of course, we have used $f_{c}(x, \alpha)=-\varepsilon^{2}+x-f(x, \alpha) \leq x-x^{2}$. Last, to show (4.3a) valid for $K_{0}-M-1 \leq l \leq K_{1}(\alpha)-M-1$, we also have, similarly to (4.6a), that

$$
D x^{l}=\prod_{n=0}^{l-1}\left[1-f_{x}\left(x^{n}, \alpha\right)\right] \leq \prod_{n=0}^{l-1}\left[1-\frac{f\left(x^{n}, \alpha\right)}{x^{n}}\right]:=I_{l},
$$

since $f_{x}(x, \alpha) \geq f(x, \alpha) / x$. On the other hand, like (4.6b), we have

$$
\begin{aligned}
x^{l} & =-\varepsilon^{2}+\left[1-\frac{f\left(x^{l-1}, \alpha\right)}{x^{l-1}}\right] x^{l-1} \\
& =\cdots=-\varepsilon^{2}\left[1+\left(1-\frac{f\left(x^{l-1}, \alpha\right)}{x^{l-1}}\right)+\cdots+\left(1-\frac{f\left(x^{1}, \alpha\right)}{x^{1}}\right)\right]+I_{l} x^{0} .
\end{aligned}
$$

Since $K_{1}=O(1 / \varepsilon)$ and $f(x, \alpha) / x \geq-1$ for $-\delta<x<0$,

$$
\begin{aligned}
I_{l} & =\frac{1}{x^{0}}\left\{x^{l}+\varepsilon^{2}\left[1+\left(1-\frac{f\left(x^{l-1}, \alpha\right)}{x^{l-1}}\right)+\cdots+\left(1-\frac{f\left(x^{1}, \alpha\right)}{x^{1}}\right)\right]\right\} \\
& \leq \frac{1}{x^{0}}\left[x^{l}+\varepsilon^{2} \cdot 2 l\right] \leq \frac{1}{x^{0}}\left[x^{l}+2 \varepsilon^{2}\left(K_{1}-M-1\right)\right] \\
& \leq \frac{\delta_{1}}{x_{0}-\delta_{0}}+O(\varepsilon)
\end{aligned}
$$

provided $x^{l} \leq \delta_{1}$, which is certainly the case when $l \geq K_{0}-M-1$. This shows (4.3a).

Proof of Theorem 1.7. As we have argued above, Proposition 4.1 and Lemma 4.2 imply all the conditions of Lemmas 2.1-2.3. Hence, when $\alpha \geq \alpha_{0}$, the same arguments for Theorems 1.3 and 1.4 apply and the topological conjugacy of $\bigcup_{n \in \mathbf{Z}} F^{n}(\bar{\Omega}, \alpha)\left\{q_{+}\right\}$to $\Sigma\left(K_{0}\right)$ follows immediately.

To show the properties (a)-(e) concerning the hair $h_{z}$ attached to $z \in$ $\bigcup_{n \in \mathbf{Z}} F^{n}\left(\Omega^{-} \cup \Omega^{0}, \alpha\right)$, we use Lemma 2.2. To be precise, we may assume $z \in \Omega^{-} \cup \Omega^{0}$ up to some finite iterations. Then, by Lemma 2.2, the forward invariant set containing $z$ is homeomorphic to a $C^{r-3}$ graph in $V$ over the $\left(x_{c}, x_{s}\right)$-axis. Since the backward invariant set in the box $B(\delta)$ is precisely $x_{c} \leq q_{+}$and $x_{s}=0$ (see Figure 4.3), the invariant set can only be their intersection, which is a closed curve on the $C^{r-3}$ graph parametrized by $\delta_{1} \leq x_{c} \leq q_{+}$. It is now easy to see that properties (a)-(e) follow immediately. Using the same argument as in Theorem 1.3, it is also not hard to see (1.6) holds. When $\alpha<\alpha_{0}$, let $K_{0}$ and $K_{1}(\alpha)$ be the same as in Lemma 4.2. Then the invariant subspace $\Omega^{*}(\alpha)$ as in the theorem exists and satisfies all the properties by Lemma 2.1. The nonexistence of 1-periodic orbits of large periods $\geq C_{0} / \sqrt{\left|\alpha-\alpha_{0}\right|}$ follows from Lemma 4.2 above because the largest first returning iteration time in $H_{0}$ is at most $K_{2}(\alpha)$. Of course, the asymptotic behavior of $K_{1}(\alpha)$ also follows from Lemma 4.2.

Remark. For the asymptotic behavior of the dynamics $y^{n}=y^{n-1}-\left(y^{n-1}\right)^{2}$, we claim $\left(y^{0}-\left(y^{0}\right)^{2}\right) / n \leq y^{n} \leq 1 /(n+1)$, i.e., $y^{n}=O(1 / n)$. Indeed, from our 


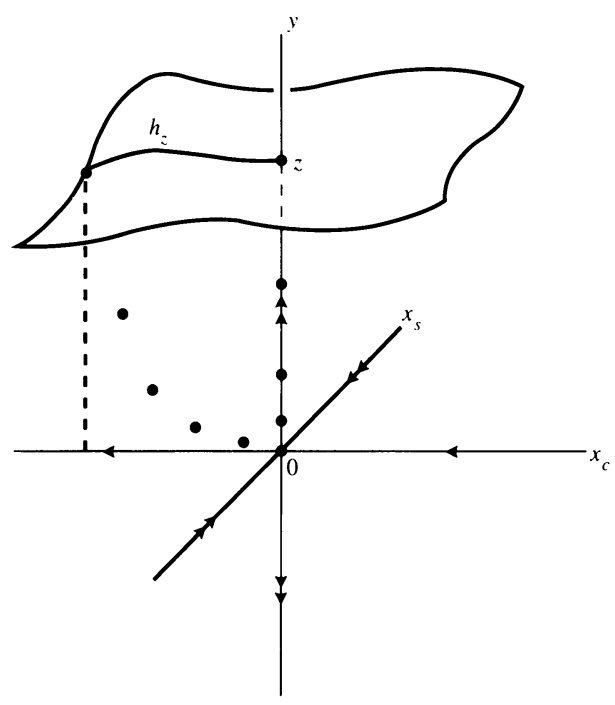

FIGURE 4.3

proof above, $0<x^{n} \leq y\left(-l, x^{n}, \alpha\right) \leq x^{n-l}$ holds for $0 \leq l \leq n$. Backwards one more step we have $0<x^{n} \leq y\left(-l, x^{n}, \alpha\right) \leq x^{n-l}$ for $0 \leq l \leq n+1$ as long as $x^{-1}$ exists. But, this is certainly the case provided that $x_{0}+\delta_{0}$ is small enough so that $f_{c}$ can be iterated backwards at least once on $B\left(x_{0}, \delta_{0}\right)$. Applying the same argument to the extended situation, we have that the denominator appearing in $y$ also remains positive. That is, $1-x^{n} \tan (n+1) \varepsilon / \varepsilon \geq 0$. In limit at $\varepsilon=0$, it reads $x^{n} \leq 1 /(n+1)$. Starting $x^{n}$ at $y^{n}$ yields $y^{n} \leq 1 /(n+1)$. (In fact, without using the limit argument, one can obtain the same inequality by going through the same comparison procedure between $\dot{y}=-y^{2}$ and $y^{n}=y^{n-1}-\left(y^{n-1}\right)^{2}$.) Using these estimates and $y^{n}=y^{n-1}-\left(y^{n-1}\right)^{2}$, it can be directly checked that $n y^{n}$ is monotone increasing. This implies $n y^{n} \geq y^{1}=$ $y^{0}-\left(y^{0}\right)^{2}$. We will refer to these estimates later in $\S 5$.

\section{Discussion}

The idea of establishing certain correspondence between invariant sets and fixed points of infinite systems is hardly new. Sil'nikov used it on several occasions. The key step in making this idea feasible is the Sil'nikov change of variables $(2.5 b)$ or $(2.5 c)$, which first systematically appeared in his works (see, e.g., Sil'nikov (1967)). Our innovation is to generalize his idea in such a way that our equations (2.8)-(2.10), in contrast to (7.1) and (7.2) of Sil'nikov (1967), are now applicable to both hyperbolic and nonhyperbolic fixed point situations. Of course, in the process, our exponential expansion Proposition 4.1 also plays a fundamental role. What is to be sketched now is the natural appearance of the cross representation $(2.5 \mathrm{~b})$ in the classical example of the Smale horseshoe map, called $f: R \rightarrow \mathbf{R}^{2}$, where $R=\{0 \leq x \leq 1,0 \leq y \leq 1\}$ is the closed unit square box. See Figure 5.1.

Let $f^{-1}(R)=H^{1} \cup H^{2}$ and $f(R) \cap R=V^{1} \cup V^{2}$ as shown. Let $f_{i}=\left.f\right|_{H^{i}}$ be the restriction on $H^{i}$. Motivated by the cross representation of (2.3) and 


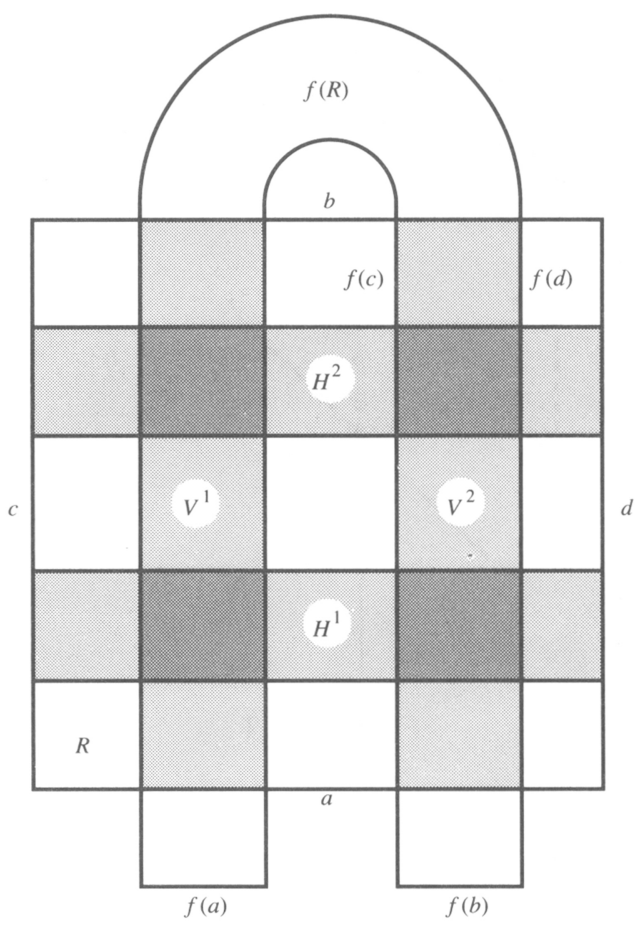

FIGURE 5.1

$(2.5 b)$, let us assume

(5.1) there exist two functions $\hat{f}_{i}=\left(\hat{f}_{i}^{x}, \hat{f}_{i}^{y}\right): R \rightarrow R$ with $\operatorname{Lip}\left(\hat{f}_{i}\right) \leq$ $\lambda<1$ for $i=1,2$ such that $\left(x^{k+1}, y^{k+1}\right)=f_{i}\left(x^{k}, y^{k}\right)$ is equivalent to

$$
y^{k}=\hat{f}_{i}^{y}\left(x^{k}, y^{k+1}\right), \quad x^{k+1}=\hat{f}_{i}^{x}\left(x^{k}, y^{k+1}\right) .
$$

Here, consistently, the superscripts refer to iterates of $f$ and $\operatorname{Lip}(g):=$ $\max \left\{\left|D_{x} g\right|+\mid D_{y} g\right\}$ for differentiable functions. Like equation (2.8), the immediate consequence is that a given orbit $\gamma=\left\{f^{k}\left(x^{0}, y^{0}\right) \in H^{s_{k}}: k \in \mathbf{Z}\right\}$ uniquely gives rise to a fixed point $\zeta:=\left(\ldots,\left(y^{k}, x^{k+1}\right), \ldots\right)$ of the following system parametrized by the sequence $s=\left(\cdots s_{-1} s_{0} s_{1} \cdots\right)$ :

$$
\begin{aligned}
x^{k} & =\hat{f}_{s_{k-1}}^{x}\left(x^{k-1}, y^{k}\right) \\
y^{k} & =\hat{f}_{s_{k}}^{y}\left(x^{k}, y^{k+1}\right) \\
x^{k+1} & =\hat{f}_{s_{k}}^{x}\left(x^{k}, y^{k+1}\right) \\
y^{k+1} & =\hat{f}_{s_{k+1}}^{y}\left(x^{k+1}, y^{k+2}\right)
\end{aligned}
$$


Give the product space $R^{\mathbf{Z}}$ a topologically equivalent metric

$$
d\left(\zeta, \zeta^{\prime}\right)=\sum_{k=-\infty}^{\infty} \frac{1}{\mu^{|k|}}\left(\left|y^{k}-y^{\prime k}\right|+\left|x^{k+1}-x^{\prime k+1}\right|\right)
$$

with $\mu>1$ chosen so that $\lambda \mu<1$. Then, by definition, it is straightforward to verify that $\lambda \mu$ is a contraction constant for the operator defined by the righthand side of the equations. Thus, the uniform contraction mapping principle again applies and the topological conjugacy between the invariant set $\Lambda(f, R)$ and $\{1,2\}^{\mathbf{Z}}$ follows immediately. Indeed, the topological conjugacy is defined naturally as $\phi(s)=\left(x^{0}(s), y^{0}(s)\right)$, where $\left(\ldots,\left(y^{-1}, x^{0}\right),\left(y^{0}, x^{1}\right), \ldots\right)(s)$ represents the fixed point, and the homeomorphic property follows from the identity

$$
\phi\left(B_{s_{-} \cdots s_{0} \cdots s_{k}}\right)=\bigcap_{i=-l}^{k} f^{-i}\left(H^{s_{i}}\right) \cap \operatorname{im} \phi .
$$

The next question one would naturally ask is, can condition (5.1) be checked easily? To this end, let us assume that the $x$-direction of $f$ is contractive and the $y$-direction is expansive. Specifically,

$$
\operatorname{Lip}\left(f_{i}^{x}\right)<\frac{1}{2} \text { and } \operatorname{Lip}\left(\left(f^{-1}\right)_{i}^{y}\right)<\frac{1}{2},
$$

where $\left(f^{-1}\right)_{i}$ is the inverse map from $V^{i}$ onto $H^{i}$. We claim that (5.3) implies (5.1). Indeed, let $(\bar{x}, \bar{y})=\left(f_{i}^{x}, f_{i}^{y}\right)(x, y)$. Then

$$
(x, y)=\left(\left(f^{-1}\right)_{i}^{x},\left(f^{-1}\right)_{i}^{y}\right)(\bar{x}, \bar{y}) .
$$

Applying substitution, we have the identity

$$
y=\left(f^{-1}\right)_{i}^{y}\left(f_{i}^{x}(x, y), \bar{y}\right) .
$$

It is easy to see that by (5.3) and the implicit function theorem (of course, $f$ is assumed $C^{1}$ as usual), $y$ can be solved as a function, say $\hat{f}_{i}^{y}$, of $x$ and $\bar{y}$. Let $\hat{f}_{i}^{x}=f_{i}^{x}\left(\cdot, \hat{f}_{i}^{y}(\cdot, \cdot)\right)$; we obtain the desired functions and it is straightforward to check (5.1) by the implicit function theorem.

As mentioned earlier in the introduction, the horseshoe theorem in Figure 1.2(a) cannot directly apply to the saddle-node fixed point case where the uniform contraction assumption (5.2) fails on the center manifold. However, the topological argument used by, e.g., Moser (1973) can be modified to cope with this nonhyperbolicity. To see this, let us recall that for a given rightward sequence $s=\left(s_{0} s_{1} \cdots\right)$ there is associated a forward invariant set $I:=$ $\left\{z^{0}: G^{k}\left(z^{0}\right) \in H^{s_{k}}, k \geq 0\right\}$, where $G:=F^{P}$ (with $F$ and $P$ as in the introduction). Thus, $I=\bigcap_{k \geq 0} G^{-k}\left(H^{s_{k}}\right)$. Similarly, for a leftward sequence $s=\left(\cdots s_{-2} s_{-1} s_{0}\right)$ we have $J:=\left\{z^{0}: G^{k}\left(z^{0}\right) \in V^{s_{k}}, k \leq 0\right\}=\bigcap_{k \leq 0} G^{-k}\left(V^{s_{k}}\right)$. We claim that the width of all the vertical strips $G^{-k}\left(V^{s_{k}}\right), k \leq 0$, and the height of all the horizontal strips $G^{-k}\left(H^{s_{k}}\right), k \geq 0$, are of order $O(1 / k)$. Being in the noncenter direction, the estimate is trivial for all the horizontal strips. In fact, they shrink at an exponential rate. Hence, it only remains to check the width of $G^{-k}\left(V^{i}\right)$. But, in our admissible variables of Proposition 
4.1, its vertical boundaries for $G^{-k}\left(V^{i}\right) \cap V^{1}$ are more or less straight lines $x=0$ and $x^{-k}\left(x_{0}\right)=f_{c}^{-k}\left(x_{0}\right), k \leq 0$, with the same notation as in Lemma 4.2 and the remark afterwards. Thus, by the remark, the width is approximately $x^{-k}\left(x_{0}\right) \leq 1 /(|k|+1)$. Therefore, it is not hard to see the width of $G^{-k}\left(V^{i}\right) \cap V^{j}$ is of order $1 /|k|$. This completes the order estimate. Finally, as the topological argument goes, $I \cap J$ is a unique invariant point parametrized by the doubly infinite sequence $\left(\cdots s_{-1} s_{0} s_{1} \cdots\right)$. Denote this correspondence as $\phi:\{1,2\}^{\mathbf{Z}} \rightarrow R$. The identity (5.2) also holds, showing the topological conjugacy. Thus, a structurally unstable horseshoe is obtained. Similar to the hairy structure of $\Lambda\left(F\left(\cdot, \alpha_{0}\right), U\right)$ for the saddle-node case as in Theorem 1.7, one can also derive a hairy horseshoe by expanding the rectangle $R$ in Figure 1.2(a) to the left of the origin a little bit.

Speaking about the hairy structure of $\Lambda(F(\cdot, \alpha), U)$ of Theorem 1.7 for $\alpha \geq \alpha_{0}$, it is easy to see that there is a natural homotopy from the full invariant set to its subspace $\bigcup_{n \in \mathbf{Z}} F^{n}(\bar{\Omega}, \alpha) \cup\left\{q_{+}\right\}$, shrinking the hairs to their roots on $\bigcup_{n \in \mathbf{Z}} F^{n}\left(\Omega^{-} \cup \Omega^{0}, \alpha\right)$ through a deformation retraction. It in turn is homotopic to our symbolic space $\Sigma\left(K_{0}\right)$. Denote this homotopy equivalence as $\varphi$. Then an obvious property of $\varphi$ in terms of the dynamics is the commutativity: $\varphi \circ F=$ $\sigma \circ \varphi$. Indeed, this follows from the invariant property of the hair $F\left(h_{z}, \alpha\right)=$ $h_{F(z, \alpha)}$ by (a) of Theorem 1.7. We are now naturally led to the following: Two dynamical systems $\{X, f\}$ and $\{Y, g\}$ are homotopically conjugate if there is a homotopy equivalence $\varphi: X \rightarrow Y$ such that the diagram

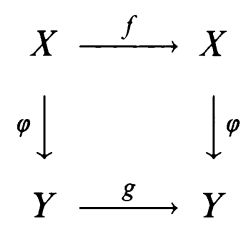

commutes. We feel that the notion of homotopy conjugacy is most appropriate and natural when the 'ordinary' bifurcations become secondary and thus negligible in regard to the onset of chaos in which they are embedded. With this new terminology, it will be a lot easier for us to describe next the dynamics $\{\Lambda(F(\cdot, \alpha), U), F(\cdot, \alpha)\}$ of other types of nonhyperbolic fixed points to which there is an irreducible and transverse homoclinic orbit.

To begin with let us point out the obvious generalization of Lemma 4.2 to the cases where, in the notation of that lemma, the center dynamics $f_{c}$ is (a) transcritical, $x+(\alpha x-f(x))$, (b) pitchfork, $x+x(\alpha-f(x))$, or (c) periodic doubling, $-x-x(\alpha-f(x))$, with $f \geq 0$ and $f(x)=O\left(x^{2}\right)$, or even more generally with an appropriate $f$ with $f(x)=O\left(x^{n}\right)$ for some finite $n \geq 2$ (cf. Guckenheimer and Holmes (1983) on those nonhyperbolic fixed points). Let us take the pitchfork case for example. Mimicking the same procedure as in the proof of Lemma 4.1, we first shift the bifurcated fixed point, say $x^{+}(\alpha)$, which is on the right side of the origin, to the origin by changing $x \rightarrow x+x^{+}$and obtain the new dynamics

$$
\bar{f}_{c}:=x+\left(x+x^{+}(\alpha)\right)\left[\alpha-f\left(x+x^{+}(\alpha), \alpha\right)\right]:=\lambda(\alpha) x-f_{1}(x, \alpha) .
$$

By direct differentiation, one can easily check that 


$$
\begin{aligned}
\lambda(\alpha) & =1-x^{+} f_{x}\left(x^{+}, \alpha\right) \leq 1, \\
f_{1}(0, \alpha) & =f_{1 x}(0, \alpha)=0, \\
f_{1 x x}(0, \alpha) & =2 f_{x}\left(x^{+}, \alpha\right)+f_{x x}\left(x^{+}, \alpha\right) x^{+} \\
& =3 f_{x x}(0, \alpha) x^{+}+O\left(\left|x^{+}\right|^{2}\right) \geq 0, \\
f_{1 x x x}(0, \alpha) & =3 f_{x x}\left(x^{+}, \alpha\right)+f_{x x x}\left(x^{+}, \alpha\right) x^{+}>0 .
\end{aligned}
$$

Similarly $x^{l}\left(x^{0}, \alpha\right)$ converges to zero uniformly for $x^{0} \in B\left(x_{0}, \delta_{0}\right)$ by the same argument, using the upper orbit $y^{l}=y^{l-1}-\left(y^{l-1}\right)^{3}$ with $y^{0}=x_{0}+\delta_{0}$. Since we also have $f_{1 x}(x) \geq f_{1}(x) / x$, the estimate (4.6a) holds. Hence (4.6b) together with $x^{l} \rightarrow 0$ uniformly implies $D x^{l} \rightarrow 0$ uniformly. Since we always have a fixed point on the center manifold in all the cases considered, we do not have to go through the second half of the proof of Lemma 4.1. Once this lemma on the convergence rate of the center flow is established, all other arguments work similarly. As a consequence of this, the fishnet will never be torn in contrast to the saddle-node case. Back to our discussion on homotopy conjugacy, we now can easily state that the dynamical systems $\{\Lambda(F(\cdot, \alpha), U), F(\cdot, \alpha)\}$ are homotopically conjugate to the shift dynamics $\left\{\Sigma\left(K_{0}\right), \sigma\right\}$ for all small $\alpha$, regardless of whether the parameter is generic or not generic to the bifurcation. In other words, $\left\{\Sigma\left(K_{0}\right), \sigma\right\}$ is homotopically invariant for (degenerate or nondegenerate) transcritical, pitchfork, and periodic doubling bifurcations of the fixed points. It is even true when the fixed point undergoes Hopf bifurcation. See Figure 5.2 for an illustrative explanation. In this sense, the saddle-nodeirreducible-homoclinic bifurcation of Theorem 1.7 is a truly genuine one in regards to either of the two notions of conjugacy equivalence.

Another way to look at these bifurcations is through tracking down all the irreducible nonwandering sets in $\Lambda_{\alpha}$. A nonwandering set $\Omega$ (excuse our slight abuse of notion here) of a map $F$ consists of points $p$ so that for every open neighborhood $U$ of $p$ there is an $n \in \mathbf{Z}$ and $F^{n}(U) \cap U \neq \varnothing$. A nonwandering set is indecomposable if it contains a dense orbit. Take, for example, the saddle-node case. At $\alpha=\alpha_{0}$ the only nonwandering set $\Omega_{0}$ is $\Lambda_{\alpha_{0}}$ itself with all the hair cut off. $\left\{\Omega_{0}, F\left(\cdot, \alpha_{0}\right)\right\}$ is nonhyperbolic but it is conjugate to $\{\Sigma, \sigma\}$ anyway. When $\alpha>\alpha_{0}, \Lambda_{\alpha}$ contains two maximal indecomposable nonwandering sets $\Omega_{0}$ and $\Omega_{1} . \Omega_{0}$ here is the stablized counterpart of $\Lambda_{\alpha_{0}}$ and $\Omega_{1}=\left\{q_{-}\right\}$is just the other bifurcated fixed point. In other words, a nonchaotic nonwandering set $\Omega_{1}$ pinches itself off from a chaotic and nonhyperbolic nonwandering set $\Omega_{0}$. Moreover, all the hair $h_{z}$ forms a dense connecting set from $\Omega_{1}$ to $\Omega_{0}$. Notice that this description is a specific local picture of the spectral decomposition of diffeomorphisms by Smale (1967). As we have seen above, when $\alpha<\alpha_{0}$ we have only partially understood the changes of $\Omega_{0}$. We end this paragraph by asking can a chaotic nonwandering set bifurcate from a chaotic and nonhyperbolic nonwandering set?

As an application, let us remark that Schecter (1987) showed that for almost all frequency $\omega$ the Poincare map (time $2 \pi / \omega$ mapping) in the phase space $(\phi, \dot{\phi})$ of the following equation

$$
\beta \ddot{\phi}+\dot{\phi}+\sin \phi=\rho+\varepsilon \sin \omega t
$$

satisfies all the conditions of Theorem 1.7 at the equilibrium point near $\phi=$ $\pi / 2, \dot{\phi}=0, \rho=1$ for some constant $\beta_{0}$ and all $0<|\varepsilon| \ll 1$. Here $\alpha=1-\rho$ 


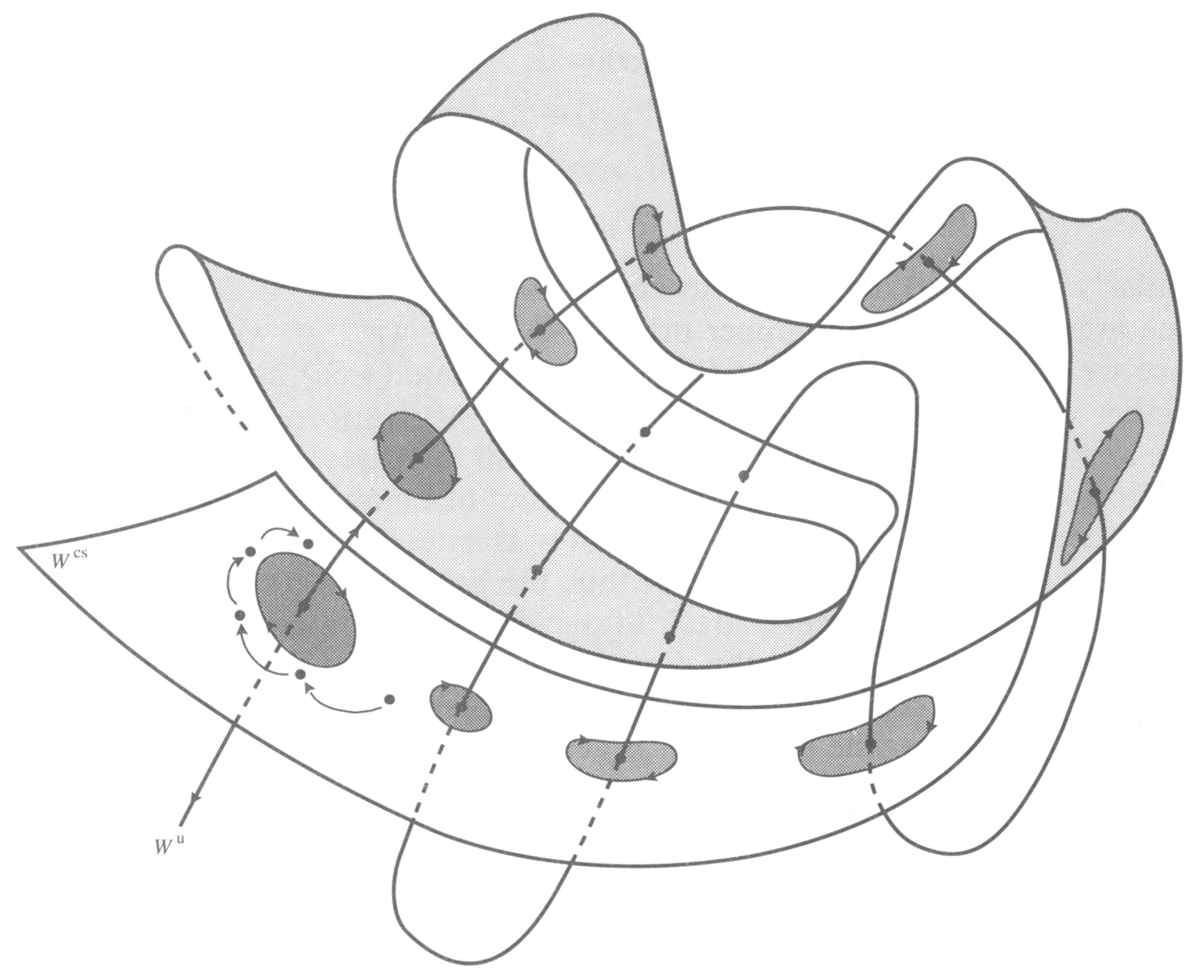

FIGURE 5.2

with $\alpha_{0}$ near 0 is the relevant parameter in terms of Theorem 1.7. This gives us the first concrete example of our net weaving bifurcation scenario. We note that this equation models the pendulum equation with linear damping $\dot{\phi}$, a constant applied torque $\rho$, and a small sinusoidal applied torque $\varepsilon \sin \omega t$. It also describes the AC-DC current-driven point Josephson junction.

Finally, we would like to point out that our complete description for $\Lambda(F, U)$ is just local in comparing with the horseshoe structure of Figure 1.2(b) which may locate outside the neighborhood $U$. We do not know at this point how the global dynamics are built upon our core dynamics $\Lambda(F, U)$. We do not know either if the irreducible homoclinic point becomes tangential. In regards to the second question, some partial results in $\mathbf{R}^{2}$ have been obtained by Gavrilov and Sil'nikov (1972, 1973), Newhouse (1974), Robinson (1983), and others. It is characterized by an infinite cascade of periodic sinks attached to an onset of chaos. Like the hairy structure of our fishnet dynamics, is the appearance of infinitely many sinks simply a secondary structure associated to a core dynamics whose homotopy dynamics is still unknown but probably not too hard to obtain?

\section{ACKNOWLEDGMENT}

The author of this work has benefitted from many stimulating discussions with J. K. Hale, N. Sternberg, K. Sakamoto, M. Kwapisz, G. Meisters, and S. Dunbar. He also thanks the referee for his many constructive and useful suggestions. 


\section{REFERENCES}

G. D. Birkhoff, Dynamical systems, Amer. Math. Soc. Colloq. Publ., Amer. Math. Soc., Providence, R.I., 1927; revised 1966; reprinted 1983.

B. Deng, Sil'nikov problem, invariant manifolds and $\lambda$-lemma, preprint, 1988a. , Homoclinic bifurcations with nonhyperbolic equilibria, SIAM J. Math. Anal. 21 (1990), 693$720,1988 \mathrm{~b}$.

N. K. Gavrilov and L. P. Sil'nikov, On the three dimensional dynamical systems close to a system with a structurally unstable homoclinic curve. I, Math. USSR-Sb. 17 (1972), 467-485; II, Math. USSR-Sb. 19 (1973), 139-156.

J. Guckenheimer and P. Holmes, Nonlinear oscillations, dynamical systems and bifurcation of vector fields, Springer-Verlag, 1983.

M. Hirsch, C. Pugh, and M. Shub, Invariant manifolds, Lecture Notes in Math., vol. 583, SpringerVerlag, 1977.

J. Moser, Stable and random motions in dynamical systems, Ann. of Math. Studies, no. 77, Princeton Univ. Press, 1973.

S. Newhouse, Diffeomorphisms with infinitely many sinks, Topology 13 (1974), 9-18.

H. Poincaré, Sur le problème des trois corps et ces équations de la dynamique, Mémoire Couronné du Prix de S. M. le Roi Oscar II, Paris, Acta Math. 13 (1890), 1-271.

C. Robinson, Bifurcations to infinitely many sinks, Comm. Math. Phys. 90 (1983), 433-459.

S. Schecter, Melnikov's method at a saddle-node and the dynamics of the forced Josephson junction, SIAM J. Math. Anal. 18 (1987), 1699-1715.

M. Shub, Global stability of dynamical systems, Springer-Verlag, 1987.

L. P. Sil'nikov, On a Poincaré-Birkhoff problem, Math. USSR-Sb. 3 (1967), 353-371.

S. Smale, Diffeomorphisms with many periodic points, Differential and Combinatorial Topology (S. S. Cairns, ed.), Princeton Univ. Press, 1963, pp. 63-80.

_, Differential dynamical systems, Bull. Amer. Math. Soc. 73 (1967), 747-817.

J. Sotomayer, Generic one-parameter families of vector fields, Inst. Hautes Études Sci. Publ. Math. 43 (1974), 5-46.

A. Vanderbauwhede and S. van Gils, Center manifold and contractions on a scale of Banach spaces, J. Funct. Anal. 17 (1987), 209-224.

S. Wiggins, Global bifurcations and chaos: Analytic methods, Springer-Verlag, 1988.

Department of Mathematics and Statistics, University of Nebraska-Lincoln, LinCOLN, NEBRASKA 68588-0323

E-mail address: deng@hoss.unl.edu 